\title{
FIELD THEORIES IN THE MODERN CALCULUS OF VARIATIONS
}

\author{
ANDRZEJ NOWAKOWSKI
}

\begin{abstract}
Two methods of construction of fields of extremals ("geodesic coverings") in the generalized problem of Bolza are given and, as a consequence, sufficient conditions for optimality in a form similar to Weierstrass' are formulated. The first field theory is an extension of Young's field theory"concourse of flights" for our problem; the other describes a nonclassical treatment of field theory which allows one to reject the "self-multiplier restriction".
\end{abstract}

1. Introduction. Consider the generalized problem of Bolza:

$$
\operatorname{minimize} J(x, u)=\int_{a}^{b} L(t, x(t), u(t)) d t+l(x(b))
$$

subject to

$$
\begin{gathered}
d x(t) / d t=f(t, x(t), u(t)) \quad \text { a.e. in }[a, b], \\
u(t) \in U(t) \quad \text { a.e. in }[a, b],
\end{gathered}
$$

$x(a)=c$, where $c$ is any fixed point in $R^{n}, x:[a, b] \rightarrow R^{n}$ is an absolutely continuous function, $u:[a, b] \rightarrow R^{m}$ is a Lebesgue measurable function (control function), $U:[a, b] \rightarrow R^{m}$ is a multifunction (i.e. $U(t)$ is a subset of $R^{m}$ for each $t$ in $[a, b]$ ), $L:[a, b] \times R^{n} \times R^{m} \rightarrow R, f:[a, b] \times R^{n} \times R^{m} \rightarrow R^{n}$, and $l$ is allowed to assume the value $+\infty$, i.e. $l: R^{n} \rightarrow R \cup\{+\infty\}$. In such a setting, this problem of Bolza unifies many problems of the calculus of variations and optimal control problems.

The existence of a solution for the generalized problem of Bolza was studied by R. T. Rockafellar in [11], necessary conditions were developed by F. H. Clarke in $[\mathbf{4}, \mathbf{5}]$ and regularity properties of solutions by F. H. Clarke and R. B. Vinter in [7]. In [13] local sufficient conditions for optimality were developed by a method which uses a canonical transformation of the "Hamiltonian inclusion". The existence of such a transformation is guaranteed by assuming the existence of an auxiliary function satisfying a certain inequality which is near the classical Jacobi conditions.

This paper aims at giving two methods of construction (from trajectories satisfying necessary conditions) of fields of extremals (often called "geodesic coverings") for our problem and, as a consequence of that, at formulating sufficient conditions for optimality in a form similar to Weierstrass'.

It is well known that in the classical setting of our problem (if we want to allow for constraints), the classical field may not, in general, exist [1]. The modern treatment of this case by Griffiths in [8] extends essentially the classical theory, however

Received by the editors November 19,1986 and, in revised form, August 10, 1987.

1980 Mathematics Subject Classification (1985 Revision). Primary 49B10.

Key words and phrases. Sufficient conditions, strong relative minimality, generalized problem of Bolza, calculus of variations, field of extremals, self-multiplier restriction. 
the smoothness and "nondegeneracy" of the problem are supposed there. Unfortunately, in modern control theory most problems do not satisfy these assumptions.

Young, in his fundamental work [12, Volume II, Chapter II], described a new type of field which he called "concourse of flights", that is more applicable to contemporary problems; he presented it in the context of an autonomous problem of least time. Yet, it still contains an undesirable multiplier restriction (strong normality assumption of the problem). He wrote [12, p. 265]: "The latter (this restriction), according to the experience of virtually everyone who has worked in the field, may well be unavoidable in a sufficiency theory", and further: "It almost seems that the extension of the standard classical method to problems with constraints necessitates this restriction".

In $\S 3$ we present Young's theory in the context of our problem (with the multiplier restriction). In $\S 4$ we describe a nonclassical treatment of field theory which joins Young's ideas and certain suggestions of Griffiths [8] and also allows one to cast off the "multiplier restriction". Thus this paper completes the program of studying the generalized problem of Bolza from the point of view of standard variational methods which are often thought of as "largely unavailable" in optimal control theory (see e.g. $[13$, p. 562]).

Since we shall base ourselves on [12], we use most of its original notions.

2. Notions and general assumptions. Let $\mathscr{L}$ be the collection of Lebesgue measurable subsets of $[a, b]$ and let $\mathscr{B}$ be Borel subsets of $R^{m} . \mathbf{L} \times \mathbf{B}$ denotes the $\sigma$-algebra of subsets of $[a, b] \times R^{m}$, generated by products of sets in $\mathscr{L}$ and $\mathscr{B}$. In order for problem (1)-(3) to make sense, we suppose the following basic hypothesis:

For each $s$ in $R^{n}$, the functions $(t, u) \rightarrow L(t, s, u),(t, u) \rightarrow f(t, s, u)$ are $\mathbf{L} \times \mathbf{B}$-measurable. There are functions $k_{1}, k_{2}$ in $L^{1}(a, b)$ such that, for $t$ in $[a, b], u$ in $U(t)$ and $s_{1}, s_{2}$ in $R^{n}$,

$$
\begin{aligned}
& \left|L\left(t, s_{1}, u\right)-L\left(t, s_{2}, u\right)\right| \leq k_{1}(t)\left|s_{1}-s_{2}\right|, \\
& \left|f\left(t, s_{1}, u\right)-f\left(t, s_{2}, u\right)\right| \leq k_{2}(t)\left|s_{1}-s_{2}\right| .
\end{aligned}
$$

The set $\left\{(t, u) \in[a, b] \times R^{m} \mid u \in U(t)\right\}$ is $\mathbf{L} \times \mathbf{B}$-measurable. The function $l$ is lower semicontinuous and not identically $+\infty$.

If $u(t)$ is a control function subject to (3) and $x(t)$ is an absolutely continuous function corresponding (by (2)) to $u(t)$ and, for them, $L(t, x(t), u(t))$ is summable and $l(x(b))$ is finite, then the pair $x(t), u(t)$ will be called admissible and $x(t)$ is an admissible trajectory.

An admissible pair $x(t), u(t)$ defined in the appropriate subinterval of $[a, b]$ with right end at $b$ will be termed a line of flight (briefly l.f.) if it satisfies the following principle (the maximum principle, see $[\mathbf{5}, \mathbf{6}]$ ): there exist along $x(t)$ a conjugate vector function $y(t)$, absolutely continuous in $t$ with values in $R^{n}$, and a number $y^{0} \leq 0$ such that $|y(t)|+\left|y^{0}\right|$ is nonvanishing and

$$
\begin{aligned}
& -\frac{d y(t)}{d t} \in y(t) \partial_{s} f(t, x(t), u(t))+y^{0} \partial_{s} L(t, x(t), u(t)) \quad \text { a.e., } \\
& \quad \begin{array}{l}
y(t) f(t, x(t), u(t))+y^{0} L(t, x(t), u(t)) \\
\quad=\sup \left\{y(t) f(t, x(t), u)+y^{0} L(t, x(t), u) \mid u \in U(t)\right\} \quad \text { a.e., }
\end{array}
\end{aligned}
$$




$$
\left(-y(b), y^{0}\right) \text { is normal to epi } l \text { at the point }(x(b), l(x(b))) .
$$

(The notation $\partial_{s} f$ refers to the generalized Jacobian of the function $s \rightarrow f(t, s, u(t)$ ) and, similarly, for $\partial_{s} L$ (see $[\mathbf{3}, \mathbf{6}]$ ); epi $l$ means the epigraph of $l ; y \partial_{s} f$ is matrix multiplication; relation (6) is a general form of a transversality condition).

We term a canonical line of flight (briefly c.l.f.), a trio $x(t), y(t), u(t)$ of functions and a number $y^{0}$ such that $x(t), u(t)$ define l.f. and $y(t), y^{0}$ are the corresponding conjugate function and the number $y^{0}$ satisfying (4)-(6). In a usual way we define an open arc of l.f. or of c.l.f.

The reader who wants to avoid problems of nonsmooth analysis, which are not an essential subject here, may read this paper under the additional assumption that the functions $s \rightarrow L(t, s, u), s \rightarrow f(t, s, u)$ are $C^{1}$. Then the sets $\partial_{s} L$ and $\partial_{s} f$ are single-element sets whose elements are the derivatives $L_{s}$ and $f_{s}$, respectively.

To study any family of arcs of l.f. depending on a parameter $\sigma$, let us define on an open set $G \subset R^{p}$ ( $R^{p}$ may be of some other dimension than $R^{n}$ ) a pair of continuous functions $t^{-}(\sigma), t^{+}(\sigma), a \leq t^{-}(\sigma)<t^{+}(\sigma) \leq b, \sigma \in G$. We assume that $t^{+}(\sigma)$ is $C^{1}$ in $G$. We further suppose that $G$ is a projection of a certain set $\widetilde{G} \subset R^{p+k}, k>0$, whose elements will be denoted by $(\sigma, \rho)$. $\tilde{G}$ does not have to be necessarily open; instead of that, we assume that the operation of projection is standard (see [12, p. 266]). Let $S^{-}=\left\{(t, \sigma) \mid t=t^{-}(\sigma) \geq a, \sigma \in G\right\}, S=\left\{(t, \sigma) \mid t^{-}(\sigma)<t<t^{+}(\sigma)\right.$, $\sigma \in G\}, S^{+}=\left\{(t, \sigma) \mid t=t^{+}(\sigma) \leq b, \sigma \in G\right\},[S]=S^{-} \cup S \cup S^{+}$. Similarly, we denote by $S^{*-}, S^{*}, S^{*+}$ the sets of $(t, \sigma, \rho)$ for which $t$ satisfies the same conditions as in $S^{-}, S, S^{+}$, respectively, and $(\sigma, \rho) \in \widetilde{G} ;\left[S^{*}\right]=S^{*-} \cup S^{*} \cup S^{*+}$.

In what follows we shall take into consideration not all l.f. but only those which are subject to certain conditions imposed upon them.

3. Young's theory of the field-concourse of flights. In this section we present Young's approach to the field from [12] for problem (1)-(3). To this effect, we assume, as is done in the classical theory of the field and in [12], the normality hypothesis:

$$
\text { the multiplier } y^{0} \text { in (4)-(6) is equal to }-1 \text {. }
$$

Further, denote by $T \subset R^{n+1}$ a set covered by graphs of trajectories of l.f. which, in the sequel, may be reduced to a smaller one. For each point $\left(t_{0}, x_{0}\right)$ in $T$, we write $I\left(t_{0}, x_{0}\right)$ for the value of

$$
\int_{t_{0}}^{b} L\left(t, x^{0}(t), u^{0}(t)\right) d t+l\left(x^{0}(b)\right)
$$

where $x^{0}(t), u^{0}(t)$ is an l.f. such that $x^{0}\left(t_{0}\right)=x_{0}$. In general, the map $(t, x) \rightarrow$ $I(t, x)$ in $T$ may be a multifunction; this is why we exclude from our further considerations (see [12, p. 266]) those l.f. which do not admit of the following hypothesis:

$$
\text { the map }(t, x) \rightarrow I(t, x) \text { is single-valued in } T \text {. }
$$

We shall say that a rectifiable curve $C$ lying in $T$ is bounded if $I(t, x)$ is bounded on the graph of $C$, i.e. "along" $C$.

For $\left(t_{0}, x_{0}\right)$ in $T, Y\left(t_{0}, x_{0}\right), U\left(t_{0}, x_{0}\right)$ denote the sets of values of all those $y(t), u(t)$ at $t_{0}$ for which $x(t), y(t), u(t)$ is a c.l.f. and $x\left(t_{0}\right)=x_{0}$. It is natural to expect that 
$Y(t, x)$ and $U(t, x),(t, x) \in T$, may not be single-valued. Hence, by an admissible pair of functions

$$
y(t, x) \in Y(t, x), \quad u(t, x) \in U(t, x), \quad(t, x) \in T,
$$

we shall mean single-valued functions $y(t, x), u(t, x)$ in $T$ such that, for each $\left(t_{0}, x_{0}\right)$ $\in T$ there is a c.l.f. $x(t), y(t), u(t)$ for which $x\left(t_{0}\right)=x_{0}, y\left(t_{0}, x\left(t_{0}\right)\right)=y\left(t_{0}\right)$, $u\left(t_{0}, x\left(t_{0}\right)\right)=u\left(t_{0}\right)$.

Up to now, the basic tool for studying old and new "fields" has been the Hilbert integral in its old and new forms (see e.g. [12, Volume I, Chapter I, Volume II, Chapter II; 10]). Thus, on the class of bounded rectifiable curves $C$ lying in $T$ and for admissible pairs of functions (8) such that

$$
\left[\{L(t, x, u(t, x))-y(t, x) f(t, x, u(t, x))\} \frac{d t}{d s}+y(t, x) \frac{d x}{d s}\right]
$$

are measurable functions of the arc length $s$ along $C$, we define the functional (called the Hilbert integral) by the curvilinear integral

(9)

$$
\begin{aligned}
\int_{C}\{L(t, x, u(t, x))-y(t, x) f(t, x, u(t, x))\} d t+y(t, x) d x \\
=\int_{0}^{s_{C}}[\{L(t(s), x(s), u(t(s), x(s))) \\
\left.\quad-y(t(s), x(s)) f(t(s), x(s), u(t(s), x(s)))\} \frac{d t}{d s}+y(t(s), x(s)) \frac{d x}{d s}\right] d s
\end{aligned}
$$

where $t(s), x(s), 0 \leq s \leq s_{C}$, is the description of $C$. The rest of this section will be devoted to the construction of a kind of "field" (Young's type of a field - a concourse of flights) by means of our l.f. and c.l.f. for which the Hilbert integral just defined does not depend on the choice of admissible pairs (8) and curves $C \subset T$ having the same ends.

Following [12, p. 271], we introduce a notion of an exact integrability of a set or simply of an exact set. A subset $\dot{T}$ of $T$ will be called an exact set if, for each bounded rectifiable curve $C \subset \dot{T}$ with end points $\left(t_{1}, x_{1}\right),\left(t_{2}, x_{2}\right)$, having the property that the expression $[\{L-y f\} d t / d s+y d x / d s]$ at almost every point of $C$ takes the same value for all admissible pairs of (8), we have

$$
\begin{aligned}
\int_{C}\{ & L(t, x, u(t, x))-y(t, x) f(t, x, u(t, x))\} d t+y(t, x) d x \\
& =I\left(t_{1}, x_{1}\right)-I\left(t_{2}, x_{2}\right)
\end{aligned}
$$

for each admissible pair $y(t, x) \in Y(t, x), u(t, x) \in U(t, x),(t, x) \in T$.

Spray of fights. First of all, we shall construct a family $\Sigma$ of arcs of l.f. depending on a parameter $\sigma$, described by functions

$$
x(t, \sigma), u(t, \sigma), \quad(t, \sigma) \in S,
$$

for which the study of the independence of the Hilbert integral is the nearest to the classical considerations. By $\Sigma^{*}$ we denote a family of arcs of c.l.f. which correspond to the $\operatorname{arcs}$ of $\Sigma$ and which are obtained by giving with functions (11) the corresponding function

$$
y(t, \sigma, \rho),(t, \sigma, \rho) \in S^{*}
$$


The definition of the functions $x(t, \sigma), y(t, \sigma, \rho)$ will be supposed extended to the sets $[S],\left[S^{*}\right]$. The sets of pairs $(t, x)$, where $x=x(t, \sigma)$ with $(t, \sigma)$ belonging to $S^{-}, S, S^{+},[S]$, will be denoted by $E^{-}, E, E^{+},[E]$, respectively, and the sets of values of triplets $(t, x(t, \sigma), y(t, \sigma, \rho))$ with $(t, \sigma, \rho)$ in $S^{*-}, S^{*}, S^{*+},\left[S^{*}\right]$ by $E^{*-}, E^{*}$, $E^{*+},\left[E^{*}\right]$, respectively.

We write (when $(t, \sigma) \in[S]$ )

$$
\widetilde{L}(t, \sigma), \tilde{f}(t, \sigma), \widetilde{I}^{+}(\sigma), \int_{t}^{b} \widetilde{L}(\tau, \sigma) d \tau, \int_{t}^{b} \widetilde{L}_{\sigma}(\tau, \sigma) d \tau
$$

for the expressions

$$
\begin{aligned}
& L(t, x(t, \sigma), u(t, \sigma)), f(t, x(t, \sigma), u(t, \sigma)), \\
& I\left(t^{+}(\sigma), x\left(t^{+}(\sigma), \sigma\right)\right), \int_{t}^{t^{+}(\sigma)} \widetilde{L}(\tau, \sigma) d \tau+\widetilde{I}^{+}(\sigma)=I(t, x(t, \sigma)), \\
& \int_{t}^{t^{+}(\sigma)} \widetilde{L}_{\sigma}(\tau, \sigma) d \tau+\widetilde{L}\left(t^{+}(\sigma), \sigma\right) t_{\sigma}^{+}(\sigma)+\widetilde{I}_{\sigma}^{+}(\sigma) .
\end{aligned}
$$

The following hypotheses with those on $t^{-}(\sigma), t^{+}(\sigma), G, \widetilde{G}$ are fundamental for our considerations and when they are satisfied, $\Sigma$ is called a spray of flights and $\Sigma^{*}$ a canonical spray of flights:

The functions $\widetilde{L}(t, \sigma), \tilde{f}(t, \sigma)$ are continuous in $[S]$; they have continuous derivatives $\widetilde{L}_{\sigma}(t, \sigma), \tilde{f}_{\sigma}(t, \sigma)$ in $[S]$ and $\partial L(t, x, u(t, \sigma)) / \partial \sigma$, $\partial f(t, x, u(t, \sigma)) / \partial \sigma$ for each fixed $(t, x)$ in $E$, satisfying at $x=$ $x(t, \sigma)$ the relations

$$
\begin{aligned}
& \frac{\partial \widetilde{L}}{\partial \sigma}=\frac{\partial L(t, x, u(t, \sigma))}{\partial \sigma}+L_{s}(t, x, u(t, \sigma)) x_{\sigma}(t, \sigma), \\
& \frac{\partial \tilde{f}}{\partial \sigma}=\frac{\partial f(t, x, u(t, \sigma))}{\partial \sigma}+f_{s}(t, x, u(t, \sigma)) x_{\sigma}(t, \sigma) ;
\end{aligned}
$$

for $(t, \sigma)$ in $S$, the functions $s \rightarrow L(t, s, u(t, \sigma)), s \rightarrow f(t, s, u(t, \sigma))$ are strongly differentiable at those $x$ for which $x=x(t, \sigma)$ (if these functions are of the class $C^{1}$, then they are strongly differentiable).

The function $y(t, \sigma, \rho)$ is continuous in $\left[S^{*}\right]$, the function $x(t, \sigma)$ is $C^{1}$ in $[S]$ and $u(t, \sigma)$ is Borel measurable in $[S]$.

The maps $S^{-} \rightarrow E^{-}, S \rightarrow E$ defined by $(t, \sigma) \rightarrow(t, x(t, \sigma))$ are descriptive (see [12, p. 266]).

Note that the assumptions which are required for the existence of the classical field in the "fixed endpoint problem" from the calculus of variations imply hypotheses (H2)-(H5) and (HN).

For $(t, x) \in[E] \subset T$, let $Y_{\Sigma}(t, x) \subset Y(t, x)$ and $U_{\Sigma}(t, x) \subset U(t, x)$ stand for the sets of values of $y(t, \sigma, \rho)$ and $u(t, \sigma)$ at those $(t, \sigma, \rho) \in\left[S^{*}\right],(t, \sigma) \in[S]$ for which $x(t, \sigma)=x$. By $y_{\Sigma}(t, x) \in Y_{\Sigma}(t, x), u_{\Sigma}(t, x) \in U_{\Sigma}(t, x)$ we denote an admissible pair of functions $y(t, x), u(t, x)$ defined in $[E]$ and term them functions relative to $\Sigma$. An 
exact subset $\dot{T} \subset[E]$ for admissible pairs $y(t, x) \in Y_{\Sigma}(t, x), u(t, x) \in U_{\Sigma}(t, x)$, $(t, x) \in[E]$, will be termed a relative exact set.

Now, we shall study a spray of flights with the help of Hilbert integral (9). Thus, assume we are given a spray of flights for which the set $E^{+}$is relative exact.

LEMMA 1 . There exists a continuous derivative $\tilde{I}_{\sigma}^{+}$in $G$, equal to $-\widetilde{L} t_{\sigma}^{+}-y x_{\sigma}$ in $S^{*+}$, i.e.

$$
\widetilde{I}_{\sigma}^{+}(\sigma)=-\widetilde{L}\left(t^{+}(\sigma), \sigma\right) t_{\sigma}^{+}(\sigma)-y\left(t^{+}(\sigma), \sigma, \rho\right) x_{\sigma}\left(t^{+}(\sigma), \sigma\right)
$$

for $(\sigma, \rho)$ in $\widetilde{G}$.

PROOF. Let $\left(t_{0}, \sigma_{0}, \rho_{0}\right)$ be any point of $S^{*+}$. Let $\Gamma$ be any sufficiently small rectifiable curve in $S^{+}$which starts from $\left(t_{0}, \sigma_{0}\right)$ and has the description $t=t^{+}\left(\sigma^{\alpha}\right)$ where $\sigma^{\alpha}$ varies from $\sigma_{0}$ to $\sigma_{1}$ along a segment parallel to the $\alpha$-axis of the $\sigma$-space. Let $C$ be the image of $\Gamma$ in $E^{+}$under the map $(t, \sigma) \rightarrow(t, x(t, \sigma))$, with ends $\left(t_{0}, x_{0}\right),\left(t_{1}, x_{1}\right)$. From the relative exactness of $E^{+}$we infer

$$
\begin{aligned}
& I\left(t_{0}, x_{0}\right)-I\left(t_{1}, x_{1}\right) \\
& =\int_{C}\left\{L\left(t, x, u_{\Sigma}(t, x)\right)-y_{\Sigma}(t, x) f\left(t, x, u_{\Sigma}(t, x)\right)\right\} d t+y_{\Sigma}(t, x) d x \\
& =\int_{\Gamma}(\widetilde{L}(t, \sigma)-y(t, \sigma, \rho(\sigma)) \tilde{f}(t, \sigma) \\
& \left.\quad \quad+y(t, \sigma, \rho(\sigma)) x_{t}(t, \sigma)\right) d t+y(t, \sigma, \rho(\sigma)) x_{\sigma}(t, \sigma) d \sigma \\
& =\widetilde{I}^{+}\left(\sigma_{0}\right)-\widetilde{I}^{+}\left(\sigma_{1}\right)
\end{aligned}
$$

where $\rho(\sigma)$ is a continuous function suitably chosen $\left(\rho_{0}=\rho\left(\sigma_{0}\right)\right)$ according to the standard projection. Since, along $\Gamma, x_{t}(t, \sigma)=\tilde{f}(t, \sigma)$, after dividing both sides of the last equality in (13) by $\left|\sigma_{0}-\sigma_{1}\right|$, we obtain

$$
\frac{1}{\left|\sigma_{0}-\sigma_{1}\right|} \int_{\Gamma}\left(\tilde{L} t_{\sigma}^{+}+y x_{\sigma}\right) d \sigma=\frac{\tilde{I}^{+}\left(\sigma_{0}\right)-\tilde{I}^{+}\left(\sigma_{1}\right)}{\left|\sigma_{0}-\sigma_{1}\right|} \text {. }
$$

By the continuity of $\widetilde{L} t_{\sigma}^{+}+y x_{\sigma}$ on $\Gamma$, there exist limits, when $\sigma_{1} \rightarrow \sigma_{0}$, of both sides of the last equality, i.e. we have $-\widetilde{I}_{\alpha}^{+}=\widetilde{L} t_{\alpha}^{+}+y x_{\alpha}$ at the point $\left(t_{0}, \sigma_{0}, \rho_{0}\right)$. Since $\Gamma$ and $\left(t_{0}, \sigma_{0}, \rho_{0}\right)$ were chosen arbitrarily, we conclude the assertion of the lemma.

LEMMA 2. Let $\Gamma$ denote any rectifiable curve in $[S]$ with $\left(t_{0}, \sigma_{0}\right)$ as the initial point and $\left(t_{1}, \sigma_{1}\right)$ as the terminal one. Then

$$
\int_{\Gamma} \widetilde{L}(t, \sigma) d t-\left(\int_{t}^{b} \widetilde{L}_{\sigma}(\tau, \sigma) d \tau\right) d \sigma=I\left(t_{0}, x\left(t_{0}, \sigma_{0}\right)\right)-I\left(t_{1}, x\left(t_{1}, \sigma_{1}\right)\right) .
$$

ProOF. Consider in $[S]$ the function

$$
Q(t, \sigma)=\int_{t_{0}}^{b} \widetilde{L}\left(\tau, \sigma_{0}\right) d \tau-\int_{t}^{b} \widetilde{L}(\tau, \sigma) d \tau
$$

which, by (H3) and Lemma 1 , has a continuous derivative in $[S]$. We easily check that $\widetilde{L} d t-\left(\int_{t}^{b} \widetilde{L}_{\sigma} d \tau\right) d \sigma$ is an exact differential in the variable $(t, \sigma)$ of the function 
$Q(t, \sigma)$. Hence we have

$$
\begin{gathered}
\int_{\Gamma} \widetilde{L} d t-\left(\int_{t}^{b} \widetilde{L}_{\sigma} d \tau\right) d \sigma=Q\left(t_{1}, \sigma_{1}\right)-Q\left(t_{0}, \sigma_{0}\right) \\
=I\left(t_{0}, x\left(t_{0}, \sigma_{0}\right)\right)-I\left(t_{1}, x\left(t_{1}, \sigma_{1}\right)\right)
\end{gathered}
$$

as asserted.

COROLLARY 1 . In the set $S^{*+}$ the quantity $y x_{\sigma}+\int_{t}^{b} \widetilde{L}_{\sigma} d \tau$ is identically zero.

PROOF. Proceeding as in the proof of Lemma 1, we obtain from (13)

$$
\begin{aligned}
& I\left(t_{0}, x\left(t_{0}, \sigma_{0}\right)\right)-I\left(t_{1}, x\left(t_{1}, \sigma_{1}\right)\right)=\int_{\Gamma} \tilde{L} d t+y x_{\sigma} d \sigma \\
& \quad=\int_{\Gamma} \widetilde{L} d t-\left(\int_{t}^{b} \widetilde{L}_{\sigma} d \tau\right) d \sigma+\int_{\Gamma}\left(y x_{\sigma}+\left(\int_{t}^{b} \widetilde{L}_{\sigma} d \tau\right)\right) d \sigma .
\end{aligned}
$$

Using Lemma 2 and continuing the proof of Lemma 1, we get the assertion of the corollary.

LEMMA 3. Let $C$ be a rectifiable curve lying, together with its terminal points, in $E^{-}$or in $E$. Then $C$ is bounded and there exist Borel measurable functions $y_{\Sigma}(t, x), u_{\Sigma}(t, x)$ along $C$, relative to $\Sigma$. Furthermore, the functions $y_{\Sigma}(t, x)$, $L\left(t, x, u_{\Sigma}(t, x)\right), f\left(t, x, u_{\Sigma}(t, x)\right)$ are bounded along it.

ProOF. By (H5), for each $(t, x)$ of $C$, there exists a neighborhood on $C$ that is the image of some curve $\Gamma$. For those $(t, x)$, we have

$$
I(t, x)=I(t, x(t, \sigma))=\int_{t}^{t^{+}(\sigma)} \widetilde{L}(\tau, \sigma) d \tau+\widetilde{I}^{+}(\sigma) .
$$

Thus, by (H3), Lemma 1 and Borel's covering theorem, we find the boundedness of $C$. The further assertions may be justified analogously as those of Lemma 25.1 of $[12$, p. 272] or by applying the measurable selection theorem from [2] to the multifunction $(t, x) \rightarrow\{(t, \sigma) \in \Gamma \mid x(t, \sigma)=x\}$ defined on $C$.

LEMMA 4. On each arc of the canonical spray of fights $\Sigma^{*}$ the expression

$$
y x_{\sigma}+\int_{t}^{b} \widetilde{L}_{\sigma} d \tau
$$

takes a constant value.

Let $C$ be any rectifiable curve contained in $E^{-}$or $E$ with the arc length description $t=t(s), x=x(s), 0 \leq s \leq s_{C}$. Then the function $I(t, x)$ restricted to $C$ becomes the function $\bar{I}(s)=I(t(s), x(s))$ of the variable $s$ in $\left[0, s_{C}\right]$, i.e. "along" $C$.

THEOREM 1. If the identity $y x_{\sigma}+\int_{t}^{b} \widetilde{L}_{\sigma} d \tau \equiv 0$ holds in $S^{*-}$ or $S^{*}$, then the function $\bar{I}(s)$ is absolutely continuous along $C$ and, for almost all $s$ in $\left[0, s_{C}\right]$,

$$
\begin{aligned}
\frac{d}{d s} \bar{I}(s)= & -\left\{L\left(t(s), x(s), u_{\Sigma}(t(s), x(s))\right)\right. \\
& \left.-y_{\Sigma}(t(s), x(s)) f\left(t(s), x(s), u_{\Sigma}(t(s), x(s))\right)\right\} \frac{d t}{d s}-y_{\Sigma}(t(s), x(s)) \frac{d x}{d s}
\end{aligned}
$$

for each admissible pair $y_{\Sigma}(t, x) \in Y_{\Sigma}(t, x), u_{\Sigma}(t, x) \in U_{\Sigma}(t, x),(t, x) \in[E]$. 
Notice that expression (15) has the form similar to the classical one and, in particular, to the original Weierstrass formula, but what is fundamentally different in it is that the functions $y_{\Sigma}(t, x), u_{\Sigma}(t, x)$ in (15) need not have unique relations to the spray parameter $\sigma$. This is exactly what allows one, already in the first step, to avoid the restriction of a one-to-one covering of $[E]$.

COROLLARY 2. Under the same assumption, the set $E^{-}$or $E$ is a relative exact set.

Although the problems considered in $[\mathbf{1 0}]$ and in this paper are a bit different, quantities (14) here and (19) in [10] are, at least in their form, the same, thus the proofs of Lemma 4, Theorem 1, and Corollary 2 are analogous to suitable proofs of Lemmas 5.2 and 5.3 in [10] and, in fact, to the proofs of Lemmas 26.1 and 25.3 from [12, pp. 272-275] (see also the proofs of Lemma $4^{\prime}$, Theorem $1^{\prime}$, and Corollary $2^{\prime}$ in $\left.\S 4\right)$.

A direct consequence of Corollary 1, Lemma 4, and Corollary 2 is

COROLlaRY 3 . The set $E^{-}$and $E$ are relative exact.

Our next step is to fit together many different sprays of flights.

Chain of flights. A finite or countable sequence of sprays of flights in $T$

$$
\Sigma_{1}, \Sigma_{2}, \ldots, \Sigma_{N}, \ldots
$$

will be termed a chain of flights and the corresponding sequence of canonical sprays a canonical chain if, for $i=1,2, \ldots, N, \ldots$, they fit together in inverse order so that the set $E_{i}^{*-}$ corresponding to $\Sigma_{i}^{*}$ contains $E_{i+1}^{*+}$ corresponding to $\Sigma_{i+1}^{*}$ (see $[\mathbf{1 2}, \mathrm{pp}$. 275, 276]). This implies that the set $E_{i+1}^{+}$of $\Sigma_{i+1}$ is relative exact for $\Sigma_{i}$ as well as for $\Sigma_{i+1}$. If $E_{1}^{+}$of $\Sigma_{1}$ happens to be a relative exact set, then all sets $E_{i}$ and $E_{i}^{-}$, $i=1,2, \ldots, N, \ldots$, are also relative exact sets and such a chain will be termed an exact chain of flights.

The sets $E_{i}^{-}$and $E_{i}$ of $\Sigma_{i}$ will be termed constituent sets of a chain and $E_{i}^{*-}, E_{i}^{*}$ of $\Sigma_{i}^{*}$ canonical constituent sets of a canonical chain.

Let $G_{1}$ be an open set of parameters $\sigma^{1}$, associated with the spray $\Sigma_{1}$. We suppose one more hypothesis satisfied:

The function $l^{+}\left(\sigma^{1}\right)=l\left(x\left(b, \sigma^{1}\right)\right)$ has a continuous derivative $l_{\sigma^{1}}^{+}$ in $G_{1}$. The map $S_{1}^{+} \rightarrow E_{1}^{+}\left(E_{1}^{+}=\left\{(b, x) \mid x=x\left(b, \sigma^{1}\right), \sigma^{1} \in G_{1}\right\}\right)$

(H6) has the following property: given any bounded rectifiable curve $C$ in $E_{1}^{+}$, there exists a rectifiable curve $\Gamma$ in $S_{1}^{+}$such that $C$ is its image under the map $(b, \sigma) \rightarrow(b, x(b, \sigma))$ and the ends of $C$ are the images of those of $\Gamma$.

A chain of flights which satisfies hypothesis (H6) will be called a distinguished chain of flights.

LEMma 5. For any distinguished chain of fights, the set $E_{1}^{+}$is relative exact.

Proof. Let $C$ be any bounded rectifiable curve in $E_{1}^{+}$with the arc length description $t=b, x=x(s), 0 \leq s \leq s_{C}$, and let $\Gamma$ be a rectifiable curve in $S_{1}^{+}$such that $C$ is its image, according to (H6). (If no such curve exists, we have nothing to prove.) From (6) we find that, for almost every $s$ in $\left[0, s_{C}\right], y_{\Sigma_{1}}(b, x) d x / d s$ has the 
same value for all $y_{\Sigma_{1}}(b, x)$ in $Y_{\Sigma_{1}}$. Proceeding similarly as in Lemma 3 , we get that there is a measurable and bounded function $y_{\Sigma_{1}}(b, x)$ along $C$. Therefore, since $d t=$ 0 in $E_{1}^{+}$, we have, for any admissible pair of functions $y_{\Sigma_{1}}(t, x), u_{\Sigma_{1}}(t, x),(t, x) \in$ $\left[E_{1}\right]$

$$
\begin{aligned}
& \int_{C} y_{\Sigma_{1}}(b, x) d x=\int_{\Gamma} y\left(b, \sigma^{1}, \rho\right) x_{\sigma^{1}}\left(b, \sigma^{1}\right) d \sigma^{1} \\
& \quad=\int_{\Gamma}\left[-\left(-y\left(b, \sigma^{1}, \rho\right),-1\right)\left(x_{\sigma^{1}}\left(b, \sigma^{1}\right), l_{\sigma^{1}}^{+}\left(\sigma^{1}\right)\right)\right] d \sigma^{1}-\int_{\Gamma} l_{\sigma^{1}}^{+}\left(\sigma^{1}\right) d \sigma^{1},
\end{aligned}
$$

where $\rho$ is a suitably chosen function of $\sigma^{1}$, according to the standard projection for a finite number of small subarcs of $\Gamma$. Again, by (6), the first integral on the righthand side of the last equality is zero. Hence we easily infer the relative exactness of the set $E_{1}^{+}$.

From Lemma 5, Corollary 3, and the definition of an exact chain of flights we obtain

THEOREM 2. Every distinguished chain of flights is an exact chain of flights.

$A$ concourse of fights. Denote by $K$ the family of all bounded rectifiable curves lying in $T$, and by $T_{n}, n=1,2, \ldots$, a finite or countable system of disjoint subsets of $T$ whose union is $T$. Of course, any $T_{n}$ should be a subset of some constituent set of a chain or a subset of a few constituent sets of different chains.

A curve $C \subset K$ will be called a fragment if its interior lies in some $T_{n}$. The class of such fragments will be denoted by $K_{0}$. We need a situation in which $K$ can be derived from $K_{0}$. To this effect, we shall need two forms of the addition of curves: fusion and embellishment, and two subtraction operations: cutting and trimming (see [12, p. 277]).

In the sequel, about $K$ and $K_{0}$ we shall assume that if a curve belongs to $K$ or $K_{0}$, then each arc of the curve, and also its inverse arc, is an element of $K$ or $K_{0}$, respectively. Moreover, we shall assume that the operations of embellishment and trimming can be carried out countably often under the restriction that from elements of $K$ we shall again obtain elements of $K$.

By means of the finite fusion and the countable embellishment, from the elements of $K_{0}$ let us compose a class $K_{1}$. From $K_{1}$ we then define a subclass $K_{2}$ of $K$ whose members are obtained by at most countable trimming. The method described by Young [12, Volume II, §28] can be applied only when $K_{2}=K$.

In such a situation, $K_{0}$ is called a repairable class of fragments, and the decomposition of the set $T$ into disjoint subsets $T_{n}$ a repairable decomposition. Then the set $T$ will be termed the unimpaired union of the sets $T_{n}$.

A concourse of flights is a finite or countable infinite system of distinguished chains of flights, such that $T$ is the unimpaired union of the constituent sets of these chains, and the set covered by graphs of canonical lines of flight, i.e. by graphs of pairs of functions $x(t), y(t)$, is the union of their canonical constituent sets.

Let $t(s), x(s), 0 \leq s \leq s_{C}$, be the arc length description of any bounded rectifiable curve $C$ in $T$. We introduce the last hypothesis in this section whose object is 
to ensure that a certain integral exists along each bounded rectifiable curve in $T$.

There exists in $T$ an admissible pair of functions $y(t, x) \in Y(t, x)$, $u(t, x) \in U(t, x),(t, x) \in T$, such that the expression

$$
\begin{aligned}
& \mid\{L(t(s), x(s), u(t(s), x(s))) \\
& \quad-y(t(s), x(s)) f(t(s), x(s), u(t(s), x(s)))\} d t / d s \\
& +y(t(s), x(s)) d x / d s \mid
\end{aligned}
$$

does not exceed along $C$ some integrable function of the arc length $s$ of $C$.

In the modern problems of the calculus of variations we are concerned with the actual problem of a minimum in the entire set $T$. Considering a spray of flights $\Sigma$, we have discussed on the model of the classical calculus of variations only what happens in a certain subset $T_{n}$ whose union turns out to be $T$. This means that we have information about the class of fragments $K_{0}$ and that we seek information about the class of our original curves $K$.

Proceeding quite similarly as in the proof of Theorem 29.1 from [12, p. 280], only changing expression (29.2) there to

$$
\begin{gathered}
\int_{C}\left\{L\left(t, x, u_{\Sigma}(t, x)\right)-y_{\Sigma}(t, x) f\left(t, x, u_{\Sigma}(t, x)\right)\right\} d t+y_{\Sigma}(t, x) d x \\
=I\left(t_{1}, x_{1}\right)-I\left(t_{2}, x_{2}\right)
\end{gathered}
$$

where $\left(t_{1}, x_{1}\right),\left(t_{2}, x_{2}\right)$ are the initial and final points of $C$ and $y_{\Sigma}(t, x), u_{\Sigma}(t, x)$ is an admissible pair of functions relative to $\Sigma$, and expression (29.3) on p. 281 to

$$
\begin{gathered}
\int_{C}\{L(t, x, u(t, x))-y(t, x) f(t, x, u(t, x))\} d t+y(t, x) d x \\
=I\left(t_{1}, x_{1}\right)-I\left(t_{2}, x_{2}\right)
\end{gathered}
$$

where $y(t, x), u(t, x)$ is now any admissible pair of functions in $T$, we obtain the following theorem and its corollary.

THEOREM 3. Assume that a concourse of flights exists and hypothesis (H7) is satisfied. Then the function $\bar{I}(s)$ is absolutely continuous in $\left[0, s_{C}\right]$ and, for almost all $s$ in $\left[0, s_{C}\right]$,

$$
\begin{aligned}
\frac{d}{d s} \bar{I}(s)=- & \{(t(s), x(s), u(t(s), x(s))) \\
& -y(t(s), x(s)) f(t(s), x(s), u(t(s), x(s)))\} \frac{d t}{d s} \\
& -y(t(s), x(s)) \frac{d x}{d s}
\end{aligned}
$$

for each admissible pair $y(t, x) \in Y(t, x), u(t, x) \in U(t, x),(t, x) \in T$.

COROLlARY 4. Suppose that the assumptions of Theorem 3 are satisfied. Then $T$ is an exact set and, in particular, relation (17) holds.

COROLlARY 5. Let us adopt the same assumptions. If $C$ is any arc of an admissible trajectory $x(t)$ under a control $u(t)$ starting at $x(a)=c$ whose graph is contained in $T$, then relation (17) is valid. 
In view of Corollary 4 , it suffices to note that, by the definition of a concourse of flights and Lemma 3, any arc of $x(t)$ is a bounded rectifiable curve as it is only a finite fusion of its subarcs which are contained in constituent sets.

As a consequence from Corollary 5 we obtain the following sufficient conditions for a strong relative minimum of $J$.

THEOREM 4. With the same assumptions, let an admissible trajectory $x^{*}(t)$, $t \in[a, b], x^{*}(a)=c$, under a control $u^{*}(t)$ be a member of our concourse. Then the pair $x^{*}(t), u^{*}(t)$ affords $J(x, u)$ a strong minimum relative to all admissible pairs $x(t), u(t), t \in[a, b], x(a)=c$, for which the graphs of $x(t)$ are contained in $T$.

Proof. Let $x(t), u(t), t \in[a, b], x(a)=c$, be any admissible pair such that the graph of $x(t)$ is contained in $T$. From the exactness of the set $T$ and from (5) we have

$$
\begin{aligned}
J\left(x^{*}, u^{*}\right)-J(x, u)=I(a, c)-J(x, u) \\
=\int_{a}^{b}[\{L(t, x(t), u(t, x(t))) \\
\quad-y(t, x(t)) f(t, x(t), u(t, x(t)))\}+y(t, x(t)) f(t, x(t), u(t))] d t \\
\quad-\int_{a}^{b} L(t, x(t), u(t)) d t \leq 0 .
\end{aligned}
$$

Hence we infer the assertion of the theorem.

REMARK. Define the value function $S(t, x)$ in the set $T$ as

$$
S(t, x)=\inf \left\{\int_{t}^{b} L(\tau, x(\tau), u(\tau)) d \tau+l(x(b))\right\}
$$

where the infimum is taken over admissible pairs $x(\tau), u(\tau), \tau \in[t, b]$, whose trajectories start at $(t, x) \in T$ and graphs are contained in $T$. By Theorem 4 , the infimum in (19) is attained and equals $I(t, x)$, thus it can be defined by relation (17). This means that the existence of value function (19) is determined by the existence of a concourse of flights. From Theorem 3 we further infer that $S(t, x)$ is an absolutely continuous function of the arc length along any bounded rectifiable curve contained in $T$. If we suppose $T$ to have interior points at which the function $S(t, x)$ is differentiable, then, by Theorem 3 , at those points $S(t, x)$ satisfies the partial differential equation

$$
S_{t}+H\left(t, x, S_{x}\right)=0
$$

where $H(t, x, y)=y f(t, x, u(t, x))-L(t, x, u(t, x))$. From the definition of $u(t, x)$, $y(t, x)$ and from (5) we also find that

$$
\min \left\{S_{t}+S_{x} f(t, x, u)-L(t, x, u) \mid u \in U(t)\right\}=0 .
$$

Formula (21) is called the partial differential equation of dynamic programming and $u(t, x)$ the optimal feedback control.

4. Nonclassical treatment of the "field" and concourse of flights. Most considerations of $\S 3$ were carried out in the $(t, x)$-space. Only a few facts concerned the $y$-space because of the occurrence of the variable $y$ in the Hilbert integral which was, however, defined in the $(t, x)$-space. This is the classical approach to 
field theory though many concepts are nonclassical; in particular, quite new is the concept of a concourse of flights which joins many (countable!) generalized classical fields - chains of flights-allowing us to account for several classical fields simultaneously appears in a natural way when we want to work with constraints, particularly with the Bolza problem (see e.g. [9, proof of Theorem 8.1, p. 35]).

In this section we present a nonclassical approach to field theory, using the beautiful concept of a concourse of flights.

First of all, putting

$$
H\left(t, x, y, y^{0}\right)=\sup \left\{y f(t, x, u)+y^{0} L(t, x, u) \mid u \in U(t)\right\}
$$

let us observe that (2) and (4) may be rewritten as

$$
\begin{gathered}
\frac{d x(t)}{d t} \in \partial_{y} H\left(t, x(t), y(t), y^{0}\right), \\
-\frac{d y(t)}{d t} \in \partial_{x} H\left(t, x(t), y(t), y^{0}\right) .
\end{gathered}
$$

In such a setting, $x(t)$ and $y(t)$ have the same preference; they simply satisfy a system of generalized Hamiltonian equations. Thus we may choose that space to handle which we prefer, and next, we should find a suitable independence integral associated with a certain canonical family of pairs $x(t), y(t)$ satisfying (23). It is obvious that we should choose that space (where more restrictions for a suitable family are needed) for manipulations, which allows us to cover a greater set in the $(t, x)$-space by graphs of trajectories $x(t)$.

If we have a "free endpoint problem", then the ends of $x(t)$ are free and the ends of $y(t)$ are subject to certain conditions, contrary to a "fixed endpoint problem". Thus we should also choose a "contrary" space in which we shall work with a "free endpoint problem" and it is the $(t, y)$-space in which we shall carry out most of our considerations from §3. In particular, we define a new independence integral. It is well known that just in the study of extremal problems with constraints there appeared multipliers. In our case (see the definition of l.f.) they are a function $y(t)$ and a number $y^{0}$. So, it is natural to extend the $(t, y)$-space mentioned above to the $\left(t, y, y^{0}\right)$-space, and thereby, to include the "troublesome multiplier" in our considerations, i.e. to cast off the "multiplier restriction".

The independence integral. In this section, similarly as in the previous one, for the investigation of any family of c.l.f., we define a certain independence integral, but now for curves lying in the $\left(t, y, y^{0}\right)$-space. To this effect, we have to add one coordinate $x^{0}$ to the $(t, x)$-space, described by the differential equation $d x^{0} / d t=$ $-L(t, x, u)$, i.e. for an admissible pair $x(t), u(t)$, we have

$$
-\frac{d x^{0}(t)}{d t}=L(t, x(t), u(t)) .
$$

The solution of this equation is defined as

$$
x^{0}(t)=\int_{t}^{b} L(\tau, x(\tau), u(\tau)) d \tau+x^{0}(b), \quad x^{0}(b)=l(x(b)) .
$$

For a given l.f. $x(t), u(t)$, we write $z(t)=\left(-x^{0}(t), x(t)\right)$ and, for a given c.l.f. $x(t), y(t), u(t), y^{0}$, we write $p(t)=\left(y^{0}, y(t)\right)$; so now, by the c.l.f. we mean the trio of functions $z(t), p(t), u(t)$. Further, denote by $P \subset R^{n+2}$ a set covered by graphs 
of $p(t)$ such that $z(t), p(t), u(t)$ is a c.l.f., which, in the sequel, may be reduced to a smaller one; let $T \subset R^{n+1}$ denote the same as in $\S 3$. If $\left(t_{0}, p_{0}\right)$ belongs to $P$, then we write $V\left(t_{0}, p_{0}\right)$ for the value of

$$
y_{0}^{0} \int_{t_{0}}^{b} L\left(t, x_{0}(t), u_{0}(t)\right) d t-x_{0}\left(t_{0}\right) y_{0}\left(t_{0}\right)+y_{0}^{0} l\left(x_{0}(b)\right)=-z_{0}\left(t_{0}\right) p_{0}\left(t_{0}\right)
$$

where $z_{0}(t)=\left(-x_{0}^{0}(t), x_{0}(t)\right), p_{0}(t)=\left(y_{0}^{0}, y_{0}(t)\right), u_{0}(t)$ is a c.l.f. such that $p_{0}\left(t_{0}\right)=$ $p_{0}$. Of course, the map $(t, p) \rightarrow V(t, p)$ in $P$ might be a multifunction, and this is why we assume the following hypothesis (compare (H2)):

$$
\begin{aligned}
& \text { the set } P \text { is such that the map }(t, p) \rightarrow V(t, p) \text { is single-valued in } \\
& P \text {. }
\end{aligned}
$$

A rectifiable curve $C$ lying in $P$ is called bounded if $V(t, p)$ is bounded along $C$. For $\left(t_{0}, p_{0}\right)$ in $P, Z\left(t_{0}, p_{0}\right)=\left(-X^{0}\left(t_{0}, p_{0}\right), X\left(t_{0}, p_{0}\right)\right), U\left(t_{0}, p_{0}\right)$ denote the sets of values of $z(t), u(t)$, respectively, at $t_{0}$ for which $z(t), p(t), u(t)$ is a c.l.f. and $p\left(t_{0}\right)=p_{0}$. For $(t, x)$ in $T, U(t, x)$ means the same as in the preceding section and $P(t, x)$ is defined analogously to $Y(t, x)$ therein, i.e. for $\left(t_{0}, x_{0}\right) \in T, P\left(t_{0}, x_{0}\right)$ denotes the set of values of $p(t)$ at $t_{0}$ for which $z(t)=\left(-x^{0}(t), x(t)\right), p(t), u(t)$ is a c.l.f. and $x\left(t_{0}\right)=x_{0}$. By an admissible pair of functions

$$
z(t, p)=\left(-x^{0}(t, p), x(t, p)\right) \in Z(t, p), \quad u(t, p) \in U(t, p),(t, p) \in P,
$$

we mean single-valued functions $z(t, p), u(t, p)$ defined in $P$ such that, for each $\left(t_{0}, p_{0}\right)$ in $P$, there exists a c.l.f. $z(t), p(t), u(t)$ for which $z\left(t_{0}, p\left(t_{0}\right)\right)=z\left(t_{0}\right), p\left(t_{0}\right)=$ $p_{0}, u\left(t_{0}, p\left(t_{0}\right)\right)=u\left(t_{0}\right)$. By an admissible pair of functions

$$
p(t, x)=\left(y^{0}(t, x), y(t, x)\right) \in P(t, x), \quad u(t, x) \in U(t, x),(t, x) \in T,
$$

we mean single-valued functions $p(t, x), u(t, x)$ defined in $T$ such that, for each $\left(t_{0}, x_{0}\right)$ in $T$, there exists a c.l.f. $z(t)=\left(-x^{0}(t), x(t)\right), p(t), u(t)$ for which $p\left(t_{0}, x\left(t_{0}\right)\right)$ $=p\left(t_{0}\right), u\left(t_{0}, x\left(t_{0}\right)\right)=u\left(t_{0}\right), x\left(t_{0}\right)=x_{0}$.

On any bounded rectifiable curve $C$ in $P$ with the arc length description $t(s)$, $p(s), 0 \leq s \leq s_{C}$, we define the curvilinear integral

$$
\begin{aligned}
\int_{C}\left\{y^{0} L(t, x(t, p), u(t, p))+y f(t, x(t, p), u(t, p))\right\} d t+z(t, p) d p \\
=\int_{0}^{s_{C}}\left(\left\{y^{0}(s) L(t(s), x(t(s), p(s)), u(t(s), p(s)))\right.\right. \\
\left.\quad+y(s) f(t(s), x(t(s), p(s)), u(t(s), p(s)))\} \frac{d t}{d s}+z(t(s), p(s)) \frac{d p}{d s}\right) d s
\end{aligned}
$$

for any admissible pair of functions (25) such that $\left(\left\{y^{0} L+y f\right\}(d t / d s)+z(d p / d s)\right)$ is a measurable function of the arc length $s$ along $C$. This integral defines the functional for the class of bounded rectifiable curves $C$ and admissible pairs of functions (25). Our task is to study its independence.

Similarly as in $\S 3$ we introduce the notion of an exact set, i.e. a subset $\dot{P}$ of $P$ is called an exact set if, for each bounded rectifiable curve $C \subset \dot{P}$ with ends 
$\left(t_{1}, p_{1}\right),\left(t_{2}, p_{2}\right)$, having the property that the expression $\left(\left\{y^{0} L+y f\right\}(d t / d s)+\right.$ $z(d p / d s))$ at almost every point of $C$ takes the same value for all admissible pairs (25), we have

$$
\begin{aligned}
& \int_{C}\left\{y^{0} L(t, x(t, p), u(t, p))+y f(t, x(t, p), u(t, p))\right\} d t+z(t, p) d p \\
& =V\left(t_{1}, p_{1}\right)-V\left(t_{2}, p_{2}\right)
\end{aligned}
$$

for each admissible pair $z(t, p) \in Z(t, p), u(t, p) \in U(t, p),(t, p) \in P$.

Since the general concept of the study of the independence of functional (26) is quite similar to Young's theory, we use the same notions of a spray of flights, a chain of flights, and a concourse of flights.

Spray of fights. Let $\Sigma$ denote (see (11)), analogously as in $\S 3$, a family of arcs described by functions

$$
z(t, \sigma), u(t, \sigma),(t, \sigma) \in S .
$$

Further, by $\Sigma^{*}$ we denote a family of arcs of c.l.f. which correspond to the arcs of $\Sigma$ and which are obtained by giving to functions (28) the corresponding function

$$
p(t, \sigma, \rho),(t, \sigma, \rho) \in S^{*} .
$$

As before, $z(t, \sigma), p(t, \sigma, \rho)$ will be supposed extended to the sets $[S],\left[S^{*}\right]$, and the sets $E^{-}, E, E^{+},[E]$ means here exactly the same as in $\S 3$. But now, $E^{*-}, E^{*}, E^{*+}$, $\left[E^{*}\right]$ will denote the sets of values of pairs $(t, p(t, \sigma, \rho))$ with $(t, \sigma, \rho)$ in $S^{*-}, S^{*}$, $S^{*+},\left[S^{*}\right]$, whereas those of pairs $(t, z(t, \sigma))$ with $(t, \sigma)$ in $S^{-}, S, S^{+},[S]$ will be $D^{-}, D, D^{+},[D]$.

We write (when $(\sigma, \rho(\sigma)) \in \widetilde{G}) \widetilde{V}^{+}(\sigma)$ for the expression

$$
V\left(t^{+}(\sigma), p\left(t^{+}(\sigma), \sigma, \rho(\sigma)\right)\right) .
$$

We assume the following hypotheses:

$\left(\mathrm{H} 3^{\prime}\right)=(\mathrm{H} 3)$.

The function $z(t, \sigma)$ is $C^{1}$ in $[S]$ and $u(t, \sigma)$ is Borel measurable in $[S]$. For given $\left(\sigma_{0}, \rho_{0}\right)$ in $\widetilde{G}$ and any small neighborhood $G_{0} \subset G$ $\left(\mathrm{H} 4^{\prime}\right) \quad$ of $\sigma_{0}$, there exists in $G_{0}$ a function $\rho(\sigma)$ such that $\rho\left(\sigma_{0}\right)=\rho_{0}$, all points $(\sigma, \rho(\sigma))$ for $\sigma \in G_{0}$ lie in $\widetilde{G}$, and $\bar{p}(t, \sigma)=p(t, \sigma, \rho(\sigma))$ is $C^{1}$ in $\left\{(t, \sigma) \mid t^{-}(\sigma) \leq t \leq t^{+}(\sigma), \sigma \in G_{0}\right\}$.

The maps $S^{*-} \rightarrow E^{*-}, S^{*} \rightarrow E^{*}$ defined by $(t, \sigma, \rho) \rightarrow(t, p(t, \sigma, \rho))$ are descriptive in the following sense: given any sufficiently small rectifiable curve $C \subset E^{*-}$ (or $\left.C \subset E^{*}\right)$ issuing from $\left(t_{0}, p\left(t_{0}, \sigma_{0}, \rho_{0}\right)\right)$, there exists a sufficiently small rectifiable curve $\Gamma \subset S^{-}($or $\Gamma \subset S)$

$\left(\mathrm{H} 5^{\prime}\right) \quad$ issuing from $\left(t_{0}, \sigma_{0}\right)$ such that each small arc of $C$ issuing from $\left(t_{0}, p\left(t_{0}, \sigma_{0}, \rho_{0}\right)\right)$ is the image under the map

$$
(t, \sigma) \rightarrow(t, p(t, \sigma, \rho(\sigma)))
$$

of a small arc of $\Gamma$ issuing from $\left(t_{0}, \sigma_{0}\right)$ where $\rho(\sigma)$ is as in $\left(\mathrm{H} 4^{\prime}\right)$. 
The maps $S^{-} \rightarrow D^{-}, S \rightarrow D$ defined by $(t, \sigma) \rightarrow(t, z(t, \sigma))$ have the following property: given any $\operatorname{arc} C_{z} \subset D^{-}$(or $\left.C_{z} \subset D\right)$ with the description $t_{1} \leq \tau \leq t_{2},\left(-x^{0}(\tau), x(\tau)\right)$ where $x(t)$ is a trajectory of an admissible pair $x(t), u(t), t \in[a, b], x(a)=c$, (H8) $x^{0}(t)=\int_{t}^{b} L(\tau, x(\tau), u(\tau)) d \tau+l(x(b))$ issuing from $\left(t_{1}, z\left(t_{1}, \sigma_{1}\right)\right)$, there exists a rectifiable curve $\Gamma \subset S^{-}$(or $\Gamma \subset S$ ) issuing from $\left(t_{1}, \sigma_{1}\right)$ such that every small arc of $C_{z}$ issuing from $\left(t_{1}, z\left(t_{1}, \sigma_{1}\right)\right)$ is the image under the map $(t, \sigma) \rightarrow(t, z(t, \sigma))$ of a small $\operatorname{arc}$ of $\Gamma$ issuing from $\left(t_{1}, \sigma_{1}\right)$.

If hypotheses $\left(\mathrm{H} 3^{\prime}\right)-\left(\mathrm{H} 5^{\prime}\right),(\mathrm{H} 8)$ with those on $t^{-}(\sigma), t^{+}(\sigma), G$ are satisfied, then $\Sigma$ will be called a spray of flights and $\Sigma^{*}$ a canonical spray of flights.

Note that these assumptions are analogous to (H3)-(H5) except the assumptions on $z(t, \sigma)$ and $p(t, \sigma, \rho)$ which are now stronger and this is still an undesirable restriction. It is so because we, in fact, still use the analogous technique of proofs as in Young's theory.

For $(t, p) \in\left[E^{*}\right] \subset P$, let $Z_{\Sigma}(t, p) \subset Z(t, p)$ and $U_{\Sigma}(t, p) \subset U(t, p)$ stand for the sets of values of $z(t, \sigma)$ and $u(t, \sigma)$ at those $(t, \sigma, \rho) \in\left[S^{*}\right]$ for which $p(t, \sigma, \rho)=p$. For $(t, x) \in[E] \subset T, P_{\Sigma}(t, x) \subset P(t, x)$ denotes the set of values of $p(t, \sigma, \rho)$ at those $(t, \sigma, \rho) \in\left[S^{*}\right]$ for which $x(t, \sigma)=x$. By $z_{\Sigma}(t, p) \in Z_{\Sigma}(t, p), u_{\Sigma}(t, p) \in U_{\Sigma}(t, p)$ and $p_{\Sigma}(t, x) \in P_{\Sigma}(t, x), u_{\Sigma}(t, x) \in U_{\Sigma}(t, x)$ we denote admissible pairs of functions $z(t, p), u(t, p)$ and $p(t, x), u(t, x)$ defined in $\left[E^{*}\right]$ and $[E]$, respectively, and term them functions relative to $\Sigma$. An exact subset $\dot{P} \subset\left[E^{*}\right]$ for $z(t, p) \in Z_{\Sigma}(t, p)$, $u(t, p) \in U_{\Sigma}(t, p),(t, p) \in\left[E^{*}\right]$, will further be termed a relative exact set.

Assume we are given a spray of flights for which the set $E^{*+}$ is relative exact. We state analogous lemmas and theorems as in Young's theory.

LEMMA $1^{\prime}$. For each $\left(\sigma_{0}, \rho_{0}\right) \in \widetilde{G}$, there are a set $G_{0} \subset G$ and a function $\rho(\sigma)$, described in $\left(\mathrm{H}^{\prime}\right)$ such that there exists a continuous derivative $\widetilde{V}_{\sigma}^{+}(\sigma)$ in $G_{0}$, equal to $-y^{0} \widetilde{L} t_{\sigma}^{+}-(x y)_{t} t_{\sigma}^{+}-z \bar{p}_{\sigma}$, i.e. for $\sigma$ in $G_{0}$ and $t=t^{+}(\sigma)$,

$$
\tilde{V}_{\sigma}^{+}(\sigma)=-y^{0}(\sigma, \rho(\sigma)) \widetilde{L}(t, \sigma) t_{\sigma}^{+}(\sigma)-\frac{d}{d t}(x(t, \sigma) y(t, \sigma, \rho(\sigma))) t_{\sigma}^{+}(\sigma)-z(t, \sigma) \bar{p}_{\sigma}(t, \sigma)
$$

where $\bar{p}(t, \sigma)=p(t, \sigma, \rho(\sigma))$.

ProOF. Let $\left(t_{0}, \sigma_{0}, \rho_{0}\right)$ be any point of $S^{*+}$. Let $\Gamma$ be the same as in the proof of Lemma 1 and let $G_{0}, \rho(\sigma)$ be as in $\left(\mathrm{H} 4^{\prime}\right)$. Now let $C$ be the image of $\Gamma$ in $E^{*+}$ under the map $(t, \sigma) \rightarrow(t, p(t, \sigma, \rho(\sigma)))$ with ends $\left(t_{0}, p_{0}\right),\left(t_{1}, p_{1}\right)$. From the relative exactness of $E^{*+}$ we get

$$
\begin{aligned}
V\left(t_{0}, p_{0}\right)-V\left(t_{1}, p_{1}\right) & \\
& =\int_{C}\left\{y^{0} L\left(t, x_{\Sigma}(t, p), u_{\Sigma}(t, p)\right)+y f\left(t, x_{\Sigma}(t, p), u_{\Sigma}(t, p)\right)\right\} d t+z_{\Sigma}(t, p) d p \\
& =\int_{\Gamma}\left(y^{0}(\sigma, \rho(\sigma)) \widetilde{L}(t, \sigma)+y(t, \sigma, \rho(\sigma)) \tilde{f}(t, \sigma)+x(t, \sigma) y_{t}(t, \sigma, \rho(\sigma))\right) d t \\
& =\tilde{V}^{+}\left(\sigma_{0}\right)-\tilde{V}^{+}\left(\sigma_{1}\right) .
\end{aligned}
$$

Proceeding analogously as in the proof of Lemma 1, we obtain the assertion of our lemma. 
LEMMA $2^{\prime}$. Let $\Gamma$ denote any small rectifiable curve in $[S]$ with $\left(t_{0}, \sigma_{0}\right)$ as the initial point and $\left(t_{1}, \sigma_{1}\right)$ as the terminal one. Then there exist a set $G_{0} \subset G$ and $\rho(\sigma)$ in $G_{0}\left(\right.$ see $\left.\left(\mathrm{H}^{\prime}\right)\right)$ such that

$$
\int_{\Gamma} \frac{d}{d t}(z(t, \sigma) \bar{p}(t, \sigma)) d t+\frac{d}{d \sigma}(z(t, \sigma) \bar{p}(t, \sigma)) d \sigma=V\left(t_{0}, \bar{p}\left(t_{0}, \sigma_{0}\right)\right)-V\left(t_{1}, \bar{p}\left(t_{1}, \sigma_{1}\right)\right)
$$

where $\bar{p}(t, \sigma)=p(t, \sigma, \rho(\sigma))$.

The proof follows directly from the definition of the function $V(t, p)$.

Similarly, as in Corollary 1, we obtain

COROLlaRY $1^{\prime}$. In the set $S^{*+}$ the quantity $p z_{\sigma}$ is identically zero.

We also have (compare to Lemma 3)

LEMMA $3^{\prime}$. Let $C$ be a rectifiable curve lying, together with its terminal points, in $E^{*-}$ or in $E^{*}$. Then $C$ is bounded and there exist Borel measurable functions $z_{\Sigma}(t, p), u_{\Sigma}(t, p)$ along $C$ relative to $\Sigma$. Furthermore, the functions $z_{\Sigma}(t, p)$, $L\left(t, x_{\Sigma}(t, p), u_{\Sigma}(t, p)\right), f\left(t, x_{\Sigma}(t, p), u_{\Sigma}(t, p)\right)$ are bounded along it.

LEMMA $4^{\prime}$. On each arc of the canonical spray of flights $\Sigma^{*}$ the expression $p z_{\sigma}$ takes a constant value.

PROOF. The proof is quite similar to that of Lemma 4. But, for the reader's convenience, we present the details. Let $\left(t^{\prime}, \sigma^{\prime}, \rho^{\prime}\right)$ be any point of $S^{*}$ and $z^{\prime}(t)=$ $\left(-x^{0^{\prime}}(t), x^{\prime}(t)\right), p^{\prime}(t)=\left(y^{0^{\prime}}, y^{\prime}(t)\right), u^{\prime}(t)$ the corresponding values of the functions $z\left(t, \sigma^{\prime}\right), p\left(t, \sigma^{\prime}, \rho^{\prime}\right), u\left(t, \sigma^{\prime}\right), t \in\left[t^{\prime}, t^{+}\left(\sigma^{\prime}\right)\right)$. Further, let $\alpha$ stand for any coordinate of the vector $\sigma \in G$. By performing in different orders the operations of integration in $t$ and differentiation in $\alpha$, on relation (2), and then differentiating in $t$, we get the following relation calculated at the point $\left(t, \sigma^{\prime}\right), t \in\left[t^{\prime}, t^{+}\left(\sigma^{\prime}\right)\right)$ :

$$
\frac{\partial}{\partial t} x_{\alpha}(t, \sigma)=\frac{\partial}{\partial \alpha} \tilde{f}(t, \sigma)
$$

and, analogously, at this point we find

$$
-\frac{\partial}{\partial t} x_{\alpha}^{0}(t, \sigma)=\frac{\partial}{\partial \alpha} \widetilde{L}(t, \sigma)
$$

From (4) and $\left(\mathrm{H}^{\prime}\right)$ we obtain at $\left(t, \sigma^{\prime}\right)$, for almost all $t$ in $\left[t^{\prime}, t^{+}\left(\sigma^{\prime}\right)\right)$,

$$
\begin{aligned}
x_{\alpha}(t, \sigma) \frac{\partial}{\partial t} y^{\prime}(t)= & -y^{\prime}(t) f_{s}\left(t, x^{\prime}(t), u^{\prime}(t)\right) x_{\alpha}(t, \sigma) \\
& -y^{0^{\prime}} L_{s}\left(t, x^{\prime}(t), u^{\prime}(t)\right) x_{\alpha}(t, \sigma)
\end{aligned}
$$

and, by the definition of $y^{0}$, we have at this point

$$
-x_{\alpha}^{0}(t, \sigma) \frac{\partial}{\partial t} y^{0^{\prime}}=0
$$

We add both sides of the last four equalities with (29) and $\left(29^{\circ}\right)$ multiplied by $y^{\prime}(t)$ and $y^{0^{\prime}}$, respectively. As a result we obtain at the same $\left(t, \sigma^{\prime}\right)$

$$
\begin{aligned}
\frac{\partial}{\partial t}\left(p^{\prime}(t) z_{\alpha}(t, \sigma)\right)= & y^{\prime}(t) \frac{\partial}{\partial \alpha}\left(\tilde{f}(t, \sigma)-f\left(t, x(t, \sigma), u^{\prime}(t)\right)\right) \\
& +y^{0^{\prime}} \frac{\partial}{\partial \alpha}\left(\tilde{L}(t, \sigma)-L\left(t, x(t, \sigma), u^{\prime}(t)\right)\right) .
\end{aligned}
$$


Hence, using $\left(\mathrm{H}^{\prime}\right)$ and next (5), we get

$$
\frac{\partial}{\partial t}\left(p^{\prime} z_{\alpha}\right)=0 \quad \text { at }\left(t, \sigma^{\prime}\right) \text { for almost all } t \text { in }\left[t^{\prime}, t^{+}\left(\sigma^{\prime}\right)\right) \text {. }
$$

Integrating this equality in the interval $\left[t^{\prime}, t^{+}\left(\sigma^{\prime}\right)\right)$, we easily conclude the proposition of the lemma.

We put $\bar{V}(s)=V(t(s), p(s))$ along any rectifiable curve $C$ in $E^{*-}$ or $E^{*}$ with the arc length description $t=t(s), p=p(s), 0 \leq s \leq s_{C}$.

THEOREM $1^{\prime}$. If the identity $p z_{\sigma} \equiv 0$ holds in $S^{*-}$ or $S^{*}$, then the function $\bar{V}(s)$ is absolutely continuous along $C$ and, for almost all $s$ in $\left[0, s_{C}\right]$,

$$
\begin{aligned}
\frac{d}{d s} \bar{V}(s)=-\left(\left\{y^{0}(s) L\left(t(s), x_{\Sigma}(t(s), p(s)), u_{\Sigma}(t(s), p(s))\right)\right.\right. & \\
& \left.+y(s) f\left(t(s), x_{\Sigma}(t(s), p(s)), u_{\Sigma}(t(s), p(s))\right)\right\} \frac{d t}{d s} \\
& \left.+z_{\Sigma}(t, p) \frac{d p}{d s}\right)
\end{aligned}
$$

for each admissible pair $z_{\Sigma}(t, p), u_{\Sigma}(t, p),(t, p) \in\left[E^{*}\right]$.

ProOF. We limit ourselves to the case when $C$ is contained in $E^{*}$. The proof of the second assertion is analogous. Let $e(s)=(d t / d s, d p / d s)$ stand for the direction of the tangent to $C$ defined for a.e. $s$ in $\left[0, s_{C}\right]$. Let $s_{0}$ be any point in $\left[0, s_{C}\right]$ such that $e(s)$ is approximately continuous at it. We set $t_{0}=t\left(s_{0}\right),\left(y_{0}^{0}, y_{0}\right)=$ $p_{0}=p\left(s_{0}\right)=\left(y^{0}\left(s_{0}\right), y\left(s_{0}\right)\right), e_{0}=e\left(s_{0}\right), \dot{t}_{0}=d t\left(s_{0}\right) / d s, \dot{p}_{0}=d p\left(s_{0}\right) / d s$. Let $z_{0}=\left(-x_{0}^{0}, x_{0}\right), u_{0}$ be any admissible vectors from the sets $Z_{\Sigma}\left(t_{0}, p_{0}\right), U_{\Sigma}\left(t_{0}, p_{0}\right)$ and let $\left(t_{0}, \sigma_{0}, \rho_{0}\right)$ be any point in $S^{*}$ for which $p\left(t_{0}, \sigma_{0}, \rho_{0}\right)=p_{0}, u\left(t_{0}, \sigma_{0}\right)=u_{0}$. We also put $f_{0}=f\left(t_{0}, x_{0}, u_{0}\right)$ and $L_{0}=L\left(t_{0}, x_{0}, u_{0}\right)$.

Denote by $\Gamma$ a rectifiable curve in $S$ such that small arcs of the curve $C$, issuing from $\left(t_{0}, p_{0}\right)$, are, in accordance with $\left(H 5^{\prime}\right)$, the images under the map $(t, \sigma) \rightarrow$ $(t, p(t, \sigma, \rho(\sigma)))$ of small arcs $\gamma$ of $\Gamma$ issuing from $\left(t_{0}, \sigma_{0}\right)$. Let now

$$
t=\bar{t}(v), \quad \sigma=\bar{\sigma}(v), \quad v \in I=[0, h],
$$

be the arc length parametric description of $\gamma$, such that the point $\left(t_{0}, \sigma_{0}\right)$ should correspond to the value of 0 . Next, define a continuous increasing function $s=s(v)$, $v \in I$, such that $s(0)=s_{0}$, which satisfies in $I$ the relations

$$
t(s(v))=\bar{t}(v), \quad p(s(v))=p(\bar{t}(v), \bar{\sigma}(v), \rho(\bar{\sigma}(v)))
$$

where $\rho(\sigma)$ is suitably chosen according to $\left(\mathrm{H}^{\prime}\right)$ and $\left(\mathrm{H} 4^{\prime}\right)$. We shall write $\bar{p}(t, \sigma)$ for $p(t, \sigma, \rho(\sigma))$.

Denote by $\Delta s$ and $\Delta \bar{V}$ the corresponding differences in $s$ and in $\bar{V}(s)$ at the ends of a small arc of $C$ issuing from $\left(t_{0}, p_{0}\right)$, being the image of $\gamma$. By assumption, $\bar{p} z_{\sigma}=0$ along $\gamma$. Hence, and from Lemma $2^{\prime}$, we conclude, taking account of $(31)$, that

$$
\begin{aligned}
&-\Delta \bar{V}=\int_{\gamma} \frac{d}{d t}(z(t, \sigma) \bar{p}(t, \sigma)) d t+\frac{d}{d \sigma}(z(t, \sigma) \bar{p}(t, \sigma)) d \sigma-\int_{\gamma} \bar{p}(t, \sigma) z_{\sigma}(t, \sigma) d \sigma \\
&=\int_{I}\left(\left\{y^{0}(\bar{\sigma}(v), \rho(\bar{\sigma}(v))) \tilde{L}(\bar{t}(v), \bar{\sigma}(v))+y(\bar{t}(v), \bar{\sigma}(v)\right.\right. \\
&\left.\rho(\bar{\sigma}(v))) \tilde{f}(\bar{t}(v), \bar{\sigma}(v))\} \frac{d t}{d s}+z(\bar{t}(v), \bar{\sigma}(v)) \frac{d p}{d s}\right) d s(v) .
\end{aligned}
$$


Since $\bar{p}(t, \sigma), \tilde{L}(t, \sigma), \tilde{f}(t, \sigma), z(t, \sigma)$ are continuous on $\gamma$ we deduce that they are bounded in $I$. This, along with (32), implies the uniform boundedness of the ratio $\Delta \bar{V} / \Delta s$ for all sufficiently small $\Delta s$. Thus the function $\bar{V}(s)$ is locally Lipschitz and, hence, absolutely continuous in $\left[0, s_{C}\right]$. This proves the first assertion of the theorem.

To prove the second, it is enough to show that

$$
\lim \frac{\Delta \bar{V}}{\Delta s}=-\left(\left\{y_{0}^{0} L_{0}+y_{0} f_{0}\right\} \dot{t}_{0}+z_{0} \dot{p}_{0}\right) \quad \text { as } \Delta s \rightarrow 0 .
$$

But this is quite analogous to the corresponding part of the proof of Lemma 25.3 in $[\mathbf{1 2}$, Volume II] if we take there

$$
\varphi=\varphi(v)=\left(\left\{y^{0} \tilde{L}+y \tilde{f}\right\} d t / d s+z d p / d s\right)-\left(\left\{y_{0}^{0} L_{0}+y_{0} f_{0}\right\} \dot{t}_{0}+z_{0} \dot{p}_{0}\right) .
$$

COROLLARY $2^{\prime}$. With the same assumption, the set $E^{*-}$ or $E^{*}$ is a relative exact set.

PROOF. For each fixed rectifiable curve $C$ lying in $E^{*-}$ or $E^{*}$, the function $\bar{V}(s)$ is absolutely continuous and satisfies (30) for all admissible pairs $z_{\Sigma}(t, p), u_{\Sigma}(t, p)$, $(t, p) \in\left[E^{*}\right]$. By Lemma $3^{\prime}$, we may integrate (30) along $C$ and, as a result, we obtain the equality which defines the relative exactness.

An immediate consequence of Corollary $1^{\prime}$, Lemma $4^{\prime}$ and Corollary $2^{\prime}$ is

COROLlARY $3^{\prime}$. The sets $E^{*-}$ and $E^{*}$ are relative exact.

By Theorem $1^{\prime}$ and Corollary $3^{\prime}$, we have information about curves in $E^{*-}$ or $E^{*}$, i.e. in the $(t, p)$-space but, in fact, we seek information about curves lying in $E^{-}$ or $E$, i.e. in the $(t, x)$-space. Thus, let $C_{z}$ denote any small arc contained in $D^{-}$(or $D)$, with the description $t_{1} \leq \tau \leq t_{2},\left(-x^{0}(\tau), x(\tau)\right)$ where $x(t)$ is a trajectory of an admissible pair $x(t), u(t), t \in[a, b], x(a)=c, x^{0}(t)=\int_{t}^{b} L(\tau, x(\tau), u(\tau)) d \tau+l(x(b))$, issuing from $\left(t_{1}, z\left(t_{1}, \sigma_{1}\right)\right)$. We also represent $C_{z}$ in terms of its arc length $s$ as $t=t(s), z=\left(x^{0}(s), x(s)\right)=z(s)$. Let further $\Gamma$ denote a rectifiable curve in $S^{-}$ (or $S$ ) such that small arcs of $C_{z}$ issuing from $\left(t_{1}, z\left(t_{1}, \sigma_{1}\right)\right)$ are, in accordance with (H8), the images under the map $(t, \sigma) \rightarrow(t, z(t, \sigma))$ of small arcs of $\Gamma$ issuing from $\left(t_{1}, \sigma_{1}\right)$. We represent $\Gamma$ in terms of its arc length $\lambda$ by functions $\bar{t}(\lambda), \bar{\sigma}(\lambda)$, so that the point $\left(t_{1}, \sigma_{1}\right)$ corresponds to $\lambda=0$. We can then define a continuous increasing function $s(\lambda)$ having its inverse $\lambda(s)$, which satisfies the relation

$$
t(s(\lambda))=\bar{t}(\lambda), \quad z(s(\lambda))=z(\bar{t}(\lambda), \bar{\sigma}(\lambda)) .
$$

In turn, let $C_{p}$ be the image under the map $(t, \sigma) \rightarrow(t, p(t, \sigma, \rho(\sigma)))$ of $\Gamma$, where $\rho(\sigma)$ is suitably chosen in accordance with ( $\left.\mathrm{H} 4^{\prime}\right)$, issuing from

$$
\left(t_{1}, \bar{p}\left(t_{1}, \sigma_{1}\right)\right)=\left(t_{1}, p\left(t_{1}, \sigma_{1}, \rho\left(\sigma_{1}\right)\right)\right)=\left(t_{1}, p_{1}\right)=\left(t_{1}, y_{1}^{0}, y_{1}\right) .
$$

We easily see that to small arcs of $\Gamma$ issuing from $\left(t_{1}, \sigma_{1}\right)$ there correspond small arcs of $C_{p}$ issuing from $\left(t_{1}, p_{1}\right)$. Thus we can express the final points of the small arcs of $C_{p}$ as a function of $s$. Denote by $\left(t_{2}, p_{2}\right)$ the terminal point of $C_{p}$ which corresponds to that of $C_{z} \cdot\left(t_{2},-x^{0}\left(t_{2}\right), x\left(t_{2}\right)\right)$.

By the same arguments as in Lemma 3 , along arc $t_{1} \leq \tau \leq t_{2}, x(\tau)$ lying in $E^{-}$(or $E$ ), there exist Borel measurable functions $p_{\Sigma}(t, x), u_{\Sigma}(t, x),(t, x) \in[E]$; moreover, the functions $p_{\Sigma}(t, x), L\left(t, x, u_{\Sigma}(t, x)\right), f\left(t, x, u_{\Sigma}(t, x)\right)$ are bounded along it. 
LEMMA 6. With the above notations, the following relation (34)

$$
\begin{aligned}
& V\left(t_{1}, p_{1}\right)-V\left(t_{2}, p_{2}\right)-x\left(t_{2}\right) y_{2}+x\left(t_{1}\right) y_{1}+x^{0}\left(t_{2}\right) y_{2}^{0}-x^{0}\left(t_{1}\right) y_{1}^{0} \\
& \quad=\int_{C_{z}}\left(y_{\Sigma}^{0}(t, x) L\left(t, x, u_{\Sigma}(t, x)\right)+y_{\Sigma}(t, x) f\left(t, x, u_{\Sigma}(t, x)\right)\right) d t-p_{\Sigma}(t, x) d z
\end{aligned}
$$

holds for each admissible pair $p_{\Sigma}(t, x), u_{\Sigma}(t, x),(t, x) \in[E]$.

PROOF. The proof is similar to the proofs of Theorem $1^{\prime}$ and Lemma 25.3 from $\left[12\right.$, p. 273]. Thus, we only sketch it. For convenience, we assume that $\left(t_{1}, z\left(t_{1}, \sigma_{1}\right)\right)$ is a point of approximate continuity of the derivative $(d t / d s, d z / d s)$ of the function $(t(s), z(s))$ Let $\gamma$ be a sufficiently small arc of $\Gamma$ issuing from $\left(t_{1}, \sigma_{1}\right)$, defined in the interval $I=\left[0, \lambda_{2}\right]$ of values of $\lambda$, i.e. the functions $\bar{t}(\lambda), \bar{\sigma}(\lambda)$ are defined in $I$. Denote by $\Delta V$ the difference in $V(t, p)$ at the ends of a small arc of $C_{p}$ issuing from $\left(t_{1}, p_{1}\right)$ and being the image of $\gamma$. Since the set $E^{*-}\left(E^{*}\right)$ is relative exact, therefore taking into account the above notations and the relations from (33), we obtain

$$
\begin{aligned}
&-\Delta V-x\left(\bar{t}\left(\lambda_{2}\right)\right) y\left(\bar{t}\left(\lambda_{2}\right), \bar{\sigma}\left(\lambda_{2}\right), \rho\left(\bar{\sigma}\left(\lambda_{2}\right)\right)\right)+x\left(t_{1}\right) y_{1} \\
&+x^{0}\left(\bar{t}\left(\lambda_{2}\right)\right) y^{0}\left(\bar{\sigma}\left(\lambda_{2}\right), \rho\left(\bar{\sigma}\left(\lambda_{2}\right)\right)\right)-x^{0}\left(t_{1}\right) y_{1}^{0} \\
&= \int_{\gamma}\left(y^{0}(\sigma, \rho(\sigma)) \tilde{L}(t, \sigma)+y(t, \sigma, \rho(\sigma)) \tilde{f}(t, \sigma)\right. \\
&\left.\quad+x(t, \sigma) y_{t}(t, \sigma, \rho(\sigma))\right) d t+z(t, \sigma) \bar{p}_{\sigma}(t, \sigma) d \sigma \\
& \quad-\int_{\gamma}\left(z_{t}(t, \sigma) \bar{p}(t, \sigma)+z(t, \sigma) \bar{p}_{t}(t, \sigma)\right) d t+\left(\bar{p}(t, \sigma) z_{\sigma}(t, \sigma)+z(t, \sigma) \bar{p}_{\sigma}(t, \sigma)\right) d \sigma \\
&=\int_{I}\left[\left(y^{0}(\bar{\sigma}(\lambda), \rho(\bar{\sigma}(\lambda))) \widetilde{L}(\bar{t}(\lambda), \bar{\sigma}(\lambda))\right.\right. \\
&\left.\quad+y(\bar{t}(\lambda), \bar{\sigma}(\lambda), \rho(\tilde{\sigma}(\lambda))) \tilde{f}(\bar{t}(\lambda), \bar{\sigma}(\lambda))) \frac{d t}{d s}-\bar{p}(\bar{t}(\lambda), \bar{\sigma}(\lambda)) \frac{d z}{d s}\right] d s(\lambda) .
\end{aligned}
$$

Proceeding quite analogously as in the corresponding part of the proof of Theorem $1^{\prime}$, we find the assertion of the lemma.

Chain of flights. The definitions of a chain of flights and a canonical chain are assumed the same as in $\S 3$ (with new meanings of a spray of flights and a canonical spray of flights).

If, in the set $S_{1}^{*+}=\left\{\left(t, \sigma^{1}, \rho^{1}\right) \mid t=t^{+}\left(\sigma^{1}\right),\left(\sigma^{1}, \rho^{1}\right) \in \widetilde{G}^{1}\right\}$ of $\Sigma_{1}^{*}\left(\widetilde{G}^{1}\right.$ is a set of parameters $\left(\sigma^{1}, \rho^{1}\right)$ associated with the canonical spray $\left.\Sigma_{1}^{*}\right)$ of a canonical chain of flights, the quantity $p z_{\sigma 1}$ is identically zero, then, by Lemma $4^{\prime}$ and Corollary $2^{\prime}$ (see Corollary $3^{\prime}$ ), the sets $E_{1}^{*-}, E_{1}^{*}$ of $\Sigma_{1}^{*}$ of the canonical chain are relative exact and, thus, all the sets $E_{i}^{*}$ and $E_{i}^{*-}$ of $\Sigma_{i}^{*}, i=2, \ldots, N, \ldots$, of this chain are also relative exact; such a canonical chain will be termed an exact canonical chain.

The sets $E_{i}^{-}$and $E_{i}$ of $\Sigma_{i}$ of a chain of flights are constituent sets here, too, but now, canonical constituent sets of a canonical chain are $E_{i}^{*-}, E_{i}^{*}$ of $\Sigma_{i}^{*}$ (defined in this section).

A canonical chain for which the set $S_{1}^{*+}$ of $\Sigma_{1}^{*}$ has the form $\left\{\left(t, \sigma^{1}, \rho^{1}\right) \mid t=\right.$ $\left.b,\left(\sigma^{1}, \rho^{1}\right) \in \widetilde{G}^{1}\right\}$ will be called a distinguished canonical chain.

LEMMA $5^{\prime}$. Given any distinguished canonical chain, the quantity $p z_{\sigma 1}$ is identically zero in the set $S_{1}^{*+}$ of $\Sigma_{1}^{*}$. 
PrOOF. It suffices to notice the smooth (by $\left.\left(\mathrm{H} 4^{\prime}\right)\right)$ function $\left(x\left(b, \sigma^{1}\right), l\left(x\left(b, \sigma^{1}\right)\right)\right)$, $\sigma^{1} \in G^{1}$, is a parametric description of the graph of the function $l(x)$ restricted to the projection of $E_{1}^{+}$of $\Sigma_{1}$ onto the $x$-space, and that the tangent space at each point of this graph is spanned by the columns of the matrix $\left(x_{\sigma^{1}}, l_{\sigma^{1}}^{+}\right)$where $l^{+}\left(\sigma^{1}\right)=l\left(x\left(b, \sigma^{1}\right)\right)$, and, at last, to use (6).

Analogously to Theorem 2 we have

THEOREM 2'. Each distinguished canonical chain is an exact canonical chain.

A concourse of flights. The general concept of a concourse of flights is here the same as in Young's theory. We only change the space in which we study exact sets. Thus, we now denote by $K$ the family of all bounded rectifiable curves lying in $P$, and by $P_{n}, n=1,2, \ldots$, a finite or countable system of disjoint subsets of $P$ whose union is $P$ and such that each $P_{n}$ should be contained in some canonical constituent set of a canonical chain.

Analogously as in $\S 3$ we define the classes of curves $K_{0}, K_{1}, K_{2}$ and the condition that $P$ is the unimpaired union of the sets $P_{n}$.

Denote by $M$ the family of all arcs of admissible trajectories $x(t), t \in[a, b]$, such that $x(a)=c$ and their graphs are contained in $T$. An arc from $M$ will be called a fragment if its interior portion lies in some $T_{n}\left(T_{n}, n=1,2, \ldots\right.$, are the same as in $\S 3)$. The class of such fragments is denoted by $M_{0}$. If the class $M$ can be derived from $M_{0}$ by a finite fusion, then the set $T$ will be termed the unimpaired union of the sets $T_{n}$.

A concourse of flights is a finite or countable infinite system of distinguished canonical chains such that $P$ is the unimpaired union of the canonical constituent sets of these chains, while $T$ is the unimpaired union of their constituent sets (i.e. the constituent sets of their projections: the chains of flights).

Let $t(s), p(s)=\left(y^{0}(s), y(s)\right), 0 \leq s \leq s_{C}$, be the arc length description of any bounded rectifiable curve $C$ in $P$. We assume

There exists in $P$ an admissible pair of functions $z(t, p) \in Z(t, p)$, $u(t, p) \in U(t, p),(t, p) \in P$, such that the expression

$$
\begin{aligned}
& \mid\left\{y^{0}(s) L(t(s), x(t(s), p(s)), u(t(s), p(s)))\right. \\
& \quad+y(s) f(t(s), x(t(s), p(s)), u(t(s), p(s)))\} \frac{d t}{d s}+z(t(s), p(s)) \frac{d p}{d s} \mid
\end{aligned}
$$

does not exceed along $C$ some integrable function of the arc length $s$ of $C$.

Proceeding similarly as in the proof of Theorem 29.1 of $[\mathbf{1 2}$, p. 280] changing suitable expressions (compare §3), we find

THEOREM $3^{\prime}$. Assume that a concourse of fights exists and hypothesis $\left(\mathrm{H}^{\prime}\right)$ is satisfied. Then the function $\bar{V}(s)$ is absolutely continuous in $\left[0, s_{C}\right]$ and, for almost 
every $s$ in $\left[0, s_{C}\right]$,

(35)

$$
\begin{aligned}
\frac{d}{d s} \bar{V}(s)=-( & \left\{y^{0}(s) L(t(s), x(t(s), p(s)), u(t(s), p(s)))\right. \\
& \left.+y(s) f(t(s), x(t(s), p(s)), u(t(s), p(s)))\} \frac{d t}{d s}+z(t(s), p(s)) \frac{d p}{d s}\right)
\end{aligned}
$$

for each admissible pair $z(t, p) \in Z(t, p), u(t, p) \in U(t, p),(t, p) \in P$.

COROLLARY $4^{\prime}$. Suppose that the assumptions of Theorem $3^{\prime}$ are satisfied. Then $P$ is an exact set and, in particular, we have the relation

$$
\begin{gathered}
\int_{C}\left\{y^{0} L(t, x(t, p), u(t, p))+y f(t, x(t, p), u(t, p))\right\} d t+z(t, p) d p \\
=V\left(t_{1}, p_{1}\right)-V\left(t_{2}, p_{2}\right)
\end{gathered}
$$

where $\left(t_{1}, p_{1}\right),\left(t_{2}, p_{2}\right)$ are the initial and final points of $C$ and $z(t, p), u(t, p),(t, p) \in$ $P$, is an admissible pair of functions.

Suppose the same as above. Let $C_{x}$ denote any arc of an admissible trajectory $x(t)$ under control $u(t), t \in[a, b]$, such that $x(a)=c$ and the graph of $x(t)$ is contained in $T$. We assume $C_{x}$ defined in $\left[t_{1}, t_{2}\right] \subset[a, b]$ and, for $t$ in $\left[t_{1}, t_{2}\right]$, we set

$$
x^{0}(t)=\int_{t}^{b} L(\tau, x(\tau), u(\tau)) d \tau+l(x(b)) .
$$

Define $C_{z}$ as an arc with the description $t_{1} \leq t \leq t_{2},\left(-x^{0}(t), x(t)\right)$.

By hypothesis, there is a decomposition of $T$ into disjoint subsets $T_{n}$, each of which is a subset of every constituent set $Q$ of the chains of flights of our concourse, such that $Q$ meets $T_{n}$. We define the families $M$ and $M_{0}$ as above. Of course, our $C_{x}$ belongs to $M$. Denote further by $\bar{C}_{x}$ a subarc of $C_{x}$ defined in $\left[\bar{t}_{1}, \bar{t}_{2}\right]$ which belongs to $M_{0} ; \bar{C}_{z}$ is a subarc of $C_{z}$ corresponding to $\bar{C}_{x}$. Let $\Sigma$ be any spray of flights of one of our chains, such that $\bar{C}_{x}$ meets either the set $E^{-}$or the set $E$ of $\Sigma$, i.e. $\bar{C}_{x}$ lies in some $T_{n}$ wholly contained in $E^{-}$or in $E$. In accordance with (H8) and the considerations preceding Lemma 6 , there is a rectifiable curve $\bar{C}_{p}$ corresponding to the arc $\bar{C}_{z}$, contained in the set $E^{*-}$ or $E^{*}$ of $\Sigma^{*}$, with ends $\left(\bar{t}_{1}, \bar{p}_{1}\right),\left(\bar{t}_{2}, \bar{p}_{2}\right)$, which (by Lemma $3^{\prime}$ ) is bounded. Hence, by Lemma 6 , we have equality (34) for $\bar{C}_{z}$. The arc $C_{x}$ is a finite fusion of members of $M_{0}$, thus there is a rectifiable curve $C_{p}$ corresponding to $C_{z}$, contained in $P$, with ends $\left(t_{1}, p_{1}\right)=\left(t_{1}, y_{1}^{0}, y_{1}\right)$, $\left(t_{2}, p_{2}\right)=\left(t_{2}, y_{2}^{0}, y_{2}\right)$, which is bounded. In this manner we have proved

COROLlARY $5^{\prime}$. With the above hypotheses and notations, the relation (37)

$$
\begin{aligned}
& V\left(t_{1}, p_{1}\right)-V\left(t_{2}, p_{2}\right)-x\left(t_{2}\right) y_{2}+x\left(t_{1}\right) y_{1}+x^{0}\left(t_{2}\right) y_{2}^{0}-x^{0}\left(t_{1}\right) y_{1}^{0} \\
& =\int_{t_{1}}^{t_{2}}\left[\left(y^{0}(t, x(t)) L(t, x(t), u(t, x(t)))+y(t, x(t)) f(t, x(t), u(t, x(t)))\right)\right. \\
& \left.\quad-\left(y^{0}(t, x(t)) L(t, x(t), u(t))+y(t, x(t)) f(t, x(t), u(t))\right)\right] d t
\end{aligned}
$$

holds for some admissible pair $p(t, x), u(t, x),(t, x) \in T$.

The last corollary allows one to use the theory described in this section for establishing sufficient conditions for a relative minimum of $J$. 
THEOREM 4'. Assume that a concourse of flights exists and hypothesis $\left(\mathrm{H}^{\prime}\right)$ is satisfied. Let a trio of functions $z^{*}(t)=\left(-x^{0 *}(t), x^{*}(t)\right), p^{*}(t)=\left(y^{0 *}, y^{*}(t)\right)$, $u^{*}(t), t \in[a, b]$, such that $x^{*}(a)=c, x^{0 *}(t)=\int_{t}^{b} L\left(\tau, x^{*}(\tau), u^{*}(\tau)\right) d \tau+l\left(x^{*}(b)\right), b e$ a member of our concourse. Then the pair $x^{*}(t), u^{*}(t)$ affords a strong minimum to $-y^{0 *} J$, in fact, to

$$
-\int_{a}^{b}\left(y^{0 *} L(t, x(t), u(t))+y^{*}(t)\left(\frac{d x(t)}{d t}-f(t, x(t), u(t))\right)\right) d t-y^{0 *} l(x(b))
$$

relative to all admissible pairs $x(t), u(t), t \in[a, b], x(a)=c$, for which the graphs of $x(t)$ are contained in $T$.

PROOF. The proof follows directly from Corollary $5^{\prime}$ (equality (37) is taken with $t_{1}=a, t_{2}=b, p_{1}=p^{*}(a)$ ), from the definition of $V(t, p)$ and (5). Indeed, for any admissible pair $x(t), u(t), t \in[a, b], x(a)=c$, such that the graph of $x(t)$ lies in $T$ and for the notations corresponding to it (by Corollary $5^{\prime}$ ), we have (38)

$$
\begin{gathered}
y^{0 *} \int_{a}^{b} L\left(t, x^{*}(t), u^{*}(t)\right) d t+y^{0 *} l\left(x^{*}(b)\right)-y^{0 *} \int_{a}^{b} L(t, x(t), u(t)) d t-y^{0 *} l(x(b)) \\
=V\left(a, p^{*}(a)\right)+x(a) y^{*}(a)-x^{0}(a) y^{0 *}-V\left(b, p_{2}\right)-x(b) y_{2}+x^{0}(b) y_{2}^{0} \\
=\int_{a}^{b}\left[y^{0}(t, x(t))(L(t, x(t), u(t, x(t)))-L(t, x(t), u(t)))\right. \\
\quad+y(t, x(t))(f(t, x(t), u(t, x(t)))-f(t, x(t), u(t)))] d t \geq 0 .
\end{gathered}
$$

REMARK 1 . The expression in square brackets under the last integral in (38) is the Weierstrass excess function for the Lagrangian

$$
y^{0} L(t, x, u)+y(t)(d x / d t-f(t, x, u))
$$

(compare [12, pp. 222-224]).

REMARK 2. First of all, let us observe from (4)-(6) that if, for at least one $p=\left(y^{0}, y\right)$ in $P, y^{0} \neq 0$, then we can always make a set of values of the multiplier $y^{0}$ to have a nonempty interior in $R$ or even to be the entire half-plane in $R$. Then, from (35) we infer that there exists a partial derivative $V_{y^{0}}(t, p)$ at each $(t, p) \in P$ with $y^{0} \neq 0$, and that it is equal to $x^{0}(t, p)$; so, for any such $(t, p), X^{0}(t, p)$ is a single-element set whose element is $V_{y^{0}}(t, p)$.

Next, define the dual value function $S_{D}(t, p)$ in the set $P$ as

$$
S_{D}(t, p)=\inf \left\{-y^{0} \int_{t}^{b} L(\tau, x(\tau), u(\tau)) d \tau-y^{0} l(x(b))\right\}
$$

where the infimum is taken over admissible pairs $x(\tau), u(\tau), \tau \in[t, b]$, whose trajectories start at $x(t, p), x(t, p) \in X(t, p)$, and graphs are contained in $T$. Proceeding similarly as in the proof of Theorem $4^{\prime}$, from Corollary $5^{\prime}$ we obtain that, for each $(t, p) \in P$ the infimum in (39) is attained and equals $y^{0} x^{0}(t, p)$. This means that the existence of the dual value function $S_{D}$ is determined by the existence of a concourse of flights, thus it can be calculated from relation (37).

Now, suppose $P$ to have interior points at which the function $V(t, p)$ is differentiable; then by (35), at those points $V(t, p)$ satisfies the partial differential equation

$$
V_{t}+H\left(t, p, V_{y}\right)=0
$$


where $V_{p}(t, p)=\left(V_{y^{0}}(t, p), V_{y}(t, p)\right)=\left(x^{0}(t, p), x(t, p)\right), H(t, p, v)=y^{0} L(t, v, u(t, p))$ $+y f(t, v, u(t, p))$ and $u(t, p)$ is any element of $U(t, p)$. From the definitions of $U(t, p), X(t, p)$ and from (5) we also find that at those points

$$
\min \left\{V_{t}+y f\left(t, V_{y}, u\right)+y^{0} L\left(t, V_{y}, u\right) \mid u \in U(t)\right\}=0 .
$$

Formula (40) may be considered as the dual partial differential equation of dynamic programming and $u(t, p)$ as the dual optimal feedback control.

5. Examples. This section consists of two numerical examples.

EXAMPLE 1. Consider the optimal control problem

$$
\operatorname{minimize} \int_{-2}^{1}\left(a(t) x^{2}(t)+b(t) u^{2}(t)\right) d t-3 x^{2}(1)
$$

subject to

$$
\begin{aligned}
& \frac{d x(t)}{d t}=B(t) u(t) \quad \text { a.e. in }[-2,1], \\
& u(t) \in U(t) \quad \text { a.e. in }[-2,1], \\
& x(-2)=c,
\end{aligned}
$$

where $c$ is any fixed point in $(-1,0]$,

$$
\begin{aligned}
& a(t)=\left\{\begin{array}{ll}
12, & -1 \leq t \leq 1, \\
0, & -2 \leq t<-1,
\end{array} \quad b(t)= \begin{cases}t^{2}, & -1 \leq t \leq 1, \\
1 / r, & -2 \leq t<-1,\end{cases} \right. \\
& B(t)=\left\{\begin{array}{cc}
1, \quad-1<t \leq 1, \\
-1, & t \in I_{k 1} \cup I_{k 3} \\
0, & t \in I_{k 2} \cup\{-2\},
\end{array} \quad U(t)= \begin{cases}R, & -1 \leq t \leq 1, \\
{[0, r] \cup\{-r\},} & -2 \leq t<-1,\end{cases} \right. \\
& r \in(1,+\infty), I_{k 1}=\left(-1-1 / 2^{3 k+1},-1-1 / 2^{3 k}\right], \\
& I_{k 2}=\left(-1-1 / 2^{3 k+2},-1-1 / 2^{3 k+1}\right], I_{k 3}=\left(-1-1 / 2^{3 k+3},-1-1 / 2^{3 k+2}\right], \\
& k=0,1,2, \ldots, \bigcup_{k=0}^{\infty}\left(I_{k 1} \cup I_{k 2} \cup I_{k 3}\right)=(-2,-1] .
\end{aligned}
$$

Canonical lines of flight $x(t), y(t), u(t), y^{0}$ satisfy the following conditions:

$$
\frac{d y(t)}{d t}=2 a(t) x(t) \quad \text { a.e. }
$$

$$
\begin{aligned}
\max & \left\{y^{0} b(t) u^{2}+y(t) B(t) u+y^{0} a(t) x^{2}(t) \mid u \in U(t)\right\} \\
& =y^{0} b(t) u^{2}(t)+y(t) B(t) u(t)+y^{0} a(t) x^{2}(t), \quad \text { a.e. } \\
y(1) & =6 x(1), \quad y^{0}=-1
\end{aligned}
$$


This implies that

$$
\begin{gathered}
x\left(t, c_{1}\right)=c_{1} t^{3}, \quad y\left(t, c_{1}\right)=6 c_{1} t^{4}, \quad u\left(t, c_{1}\right)=3 c_{1} t^{2}, \quad t \in[-1-1], c_{1} \in R, \\
x\left(t, c_{2}\right)=-c_{2}^{2}, \quad y\left(t, c_{2}\right)=c_{2}^{2}, \quad u\left(t, c_{2}\right)=0, \quad t \in[-2,-1], c_{2} \in[-1,1], \\
x\left(t, c_{3}\right)=-r \int_{t}^{-1} B^{2}(s) d s+c_{3}, \quad y\left(t, c_{3}\right)=-c_{3}, \quad u\left(t, c_{3}\right)=-r B^{2}(t), \\
x\left(t, c_{4}\right)=c_{4} \int_{t}^{-1} B^{2}(s) d s+c_{4}, \quad y\left(t, c_{4}\right)=-c_{4}, \quad u\left(t, c_{4}\right)=-c_{4} r B(t), \\
t \in[-2,-1], c_{4} \in(0,1 / r), \\
x\left(t, c_{5}\right)=r \int_{t}^{-1} B^{2}(s) d s+c_{5}, \quad y\left(t, c_{5}\right)=-c_{5}, \quad u\left(t, c_{5}\right)=r B^{2}(t), \\
t \in[-2,-1], c_{5} \in[1 / r,+\infty) .
\end{gathered}
$$

Notice that the trajectories $x\left(t, c_{1}\right)$ meet at the common point $(0,0)$, yet assumption (H2) is satisfied.

Now, from the above c.l.f. we form the following canonical sprays of flights:

$$
\begin{aligned}
& \Sigma_{11}^{*}: x\left(t, \sigma_{11}\right)=\left(1+\sigma_{11}^{2}\right) t^{3}, \quad y\left(t, \sigma_{11}\right)=6\left(1+\sigma_{11}^{2}\right) t^{4}, \\
& u\left(t, \sigma_{11}\right)=3\left(1+\sigma_{11}^{2}\right) t^{2}, \quad t \in[-1,1], \sigma_{11} \in R, \\
& \Sigma_{21}^{*}: x\left(t, \sigma_{21}\right)=\sigma_{21}^{2} t^{3}, \quad y\left(t, \sigma_{21}\right)=6 \sigma_{21}^{2} t^{4}, \quad u\left(t, \sigma_{21}\right)=3 \sigma_{21}^{2} t^{2}, \\
& t \in[-1,1], \sigma_{21} \in(-1,1), \\
& \Sigma_{31}^{*}: x\left(t, \sigma_{31}\right)=-\left(1 / r+\sigma_{31}^{2}\right) t^{3}, \quad y\left(t, \sigma_{31}\right)=-6\left(1 / r+\sigma_{31}^{2}\right) t^{4}, \\
& u\left(t, \sigma_{31}\right)=3\left(1 / r+\sigma_{31}^{2}\right), \quad t \in[-1,1], \sigma_{31} \in R, \\
& \Sigma_{41}^{*}: x\left(t, \sigma_{41}\right)=\sigma_{41} t^{3}, \quad y\left(t, \sigma_{41}\right)=6 \sigma_{41} t^{4}, \quad u\left(t, \sigma_{41}\right)=3 \sigma_{41} t^{2}, \\
& t \in[-1,1], \sigma_{41} \in(0,1 / r), \\
& \Sigma_{22}^{*}: x\left(t, \sigma_{22}\right)=-\sigma_{22}^{2}, \quad y\left(t, \sigma_{22}\right)=6 \sigma_{22}^{2}, \quad u\left(t, \sigma_{22}\right)=0 \text {, } \\
& t \in[-2,-1], \sigma_{22} \in(-1,1), \\
& \Sigma_{1 k j}^{*}: x\left(t, \sigma_{1 k j}\right)=-r \int_{t}^{-1} B^{2}(s) d s-\left(1+\sigma_{1 k j}^{2}\right), \\
& y\left(t, \sigma_{1 k j}\right)=6\left(1+\sigma_{1 k j}^{2}\right), \quad u\left(t, \sigma_{1 k j}\right)=-r B^{2}(t), \\
& t \in I_{k j}, \sigma_{1 k j} \in R, k=0,1, \ldots, j=1,2,3, \\
& \Sigma_{3 k j}^{*}: x\left(t, \sigma_{3 k j}\right)=r \int_{t}^{-1} B^{2}(s) d s+\left(1 / r+\sigma_{3 k j}^{2}\right), \\
& y\left(t, \sigma_{3 k j}\right)=-6\left(1+\sigma_{3 k j}^{2}\right), \quad u\left(t, \sigma_{3 k j}\right)=r B^{2}(t), \\
& t \in I_{k j}, \sigma_{3 k j} \in R, k=0,1, \ldots, j=1,2,3, \\
& \Sigma_{4 k j}^{*}: x\left(t, \sigma_{4 k j}\right)=\sigma_{4 k j} \int_{t}^{-1} B^{2}(s) d s+\sigma_{4 k j}, \quad y\left(t, \sigma_{4 k j}\right)=-\sigma_{4 k j}, \\
& u\left(t, \sigma_{4 k j}\right)=-\sigma_{4 k j} r B(t), \quad t \in I_{k j}, \sigma_{4 k j} \in(0,1 / r), \\
& k=0,1, \ldots, j=1,2,3 \text {. }
\end{aligned}
$$


Next, we join the sprays of flights in four distinguished canonical chains of flights:

$$
\begin{aligned}
& \Sigma_{21}^{*}, \Sigma_{22}^{*}, \\
& \Sigma_{11}^{*}, \Sigma_{101}^{*}, \Sigma_{102}^{*}, \Sigma_{103}^{*}, \Sigma_{111}^{*}, \Sigma_{112}^{*}, \ldots, \\
& \Sigma_{31}^{*}, \Sigma_{301}^{*}, \Sigma_{302}^{*}, \Sigma_{303}^{*}, \ldots, \\
& \Sigma_{41}^{*}, \Sigma_{401}^{*}, \Sigma_{402}^{*}, \ldots
\end{aligned}
$$

These chains form a concourse of flights (we easily check that hypotheses (H3)- $(\mathrm{H} 7)$ are fulfilled, and that a set $T$ covered by the graphs of l.f. is the unimpaired union of the constituent sets of these chains).

Thus the pair

$$
x(t)=\left\{\begin{array}{ll}
c, & t \in[-2,-1], \\
-c t^{3}, & t \in[-1,1],
\end{array} \quad u(t)= \begin{cases}0, & t \in[-2,-1), \\
-3 c t^{2}, & t \in[-1,1]\end{cases}\right.
$$

affords (41) a strong minimum relative to all admissible pairs $x(t), u(t), t \in[-2,1]$, $x(-2)=c$, for which the graphs of $x(t)$ are contained in $T$.

EXAMPLE 2. Consider the optimal control problem

$$
\operatorname{minimize} \int_{-1}^{\pi}\left(a(t) x^{2}(t)+b(t) u^{2}(t)\right) d t
$$

subject to

$$
\begin{aligned}
& \frac{d x(t)}{d t}=B(t) u(t) \quad \text { a.e. in }[-1, \pi], \\
& u(t) \in U(t) \quad \text { a.e. in }[-1, \pi], \\
& x(-1)=x(\pi)=0,
\end{aligned}
$$

where

$$
\begin{aligned}
& a(t)=\left\{\begin{array}{ll}
-1 / 2, & 0 \leq t \leq \pi, \\
0, & -1 \leq t<0,
\end{array} \quad b(t)= \begin{cases}1 / 2, & 0 \leq t \leq \pi, \\
1, & -1 \leq t<0,\end{cases} \right. \\
& B(t)= \begin{cases}1, & 0<t \leq \pi, \\
-1, & t \in I_{k 1} \cup I_{k 3}, \\
0, & t \in I_{k 2} \cup\{-1\},\end{cases} \\
& U(t)=\left\{\begin{array}{ll}
{[-1,1],} & 0 \leq t \leq \pi, \\
{[-r, r],} & -1 \leq t<0,
\end{array} \quad r \geq 0,\right. \\
& I_{k j}=\left(-1 / 2^{3 k+1},-1 / 2^{3 k+j-1}\right], \\
& \bigcup_{k=0}^{\infty} \bigcup_{j=1}^{3} I_{k j}=(-1,0] .
\end{aligned}
$$

C.l.f. $x(t), y(t), u(t), y^{0}$ satisfy (42), (43) with new $a(t), b(t), B(t), U(t)$ and $y(\pi) \in$ $R,-y^{0} \in[0,+\infty),|y(\pi)|+y^{0} \neq 0$. Hence we calculate that

$$
\begin{gathered}
y^{0}=-1, \quad x\left(t, c_{1}\right)=c_{1} \sin t, \quad y\left(t, c_{1}\right)=c_{1} \cos t, \\
u\left(t, c_{1}\right)=c_{1} \cos t, \quad t \in[0, \pi], c_{1} \in(-1,1), \\
y^{0}=-1, \quad x\left(t, c_{2}\right)=\operatorname{sign} c_{2} c_{2} \sin t, \quad y\left(t, c_{2}\right)=\operatorname{sign} c_{2} c_{2} \cos t, \\
u\left(t, c_{2}\right)=\operatorname{sign} c_{2} c_{2} \cos t, \quad t \in\left[t\left(c_{2}\right), \pi-t\left(c_{2}\right)\right], c_{2} \in(-\pi / 2,-1] \cup[1, \pi / 2),
\end{gathered}
$$


where $t\left(c_{2}\right) \in[0, \pi / 2]$ is a solution of the equation $\sin t=t /\left|c_{2}\right|, t\left(c_{2}\right)>0$ for $\left|c_{2}\right|>1$,

$$
\begin{aligned}
& y^{0}=-1, x\left(t, c_{2}\right)=\operatorname{sign} c_{2} t, \\
& y\left(t, c_{2}\right)=\operatorname{sign} c_{2}\left(-\frac{1}{2} t^{2}+c_{2} \cos t\left(c_{2}\right)+t^{2}\left(c_{2}\right) / 2\right), \\
& u\left(t, c_{2}\right)=\operatorname{sign} c_{2}, \quad t \in\left[0, t\left(c_{2}\right)\right], c_{2} \in(-\pi / 2,-1] \cup[1, \pi / 2), \\
& y^{0}=-1, \quad x\left(t, c_{2}\right)=\operatorname{sign} c_{2}(-t+\pi), \\
& y\left(t, c_{2}\right)=\operatorname{sign} c_{2}\left(\frac{1}{2} t^{2}+\pi t+c_{2} \cos d-\frac{1}{2} d^{2}-\pi d\right), u\left(t, c_{2}\right)=-\operatorname{sign} c_{2}, \\
& t \in\left[\pi-t\left(c_{2}\right), \pi\right], c_{2} \in(-\pi / 2,-1] \cup[1, \pi / 2), d=\pi-t\left(c_{2}\right), \\
& y^{0}\left(c_{3}\right)=c_{3}, \quad x\left(t, c_{3}\right)=0, \quad y\left(t, c_{3}\right)=0 \text {, } \\
& u\left(t, c_{3}\right)=0, \quad t \in[-1, \pi], c_{3} \in(-1,0) \text {, } \\
& y^{0}=-1, \quad x\left(t, c_{4}\right)=-c_{4} \int_{t}^{0} B^{2}(s) d s, \quad y\left(t, c_{4}\right)=c_{4}, \\
& u\left(t, c_{4}\right)=c_{4} B(t), \quad t \in[-1,0],\left|c_{4}\right|<\min (1, r) .
\end{aligned}
$$

If $r<1$ (or $r=1$ ), then

$$
\begin{aligned}
y^{0}=-1, \quad x\left(t, c_{5}\right)=-\operatorname{sign} c_{5} r \int_{t}^{0} B^{2}(s) d s, \quad y\left(t, c_{5}\right)=\operatorname{sign} c_{5}\left(r+c_{5}^{2}\right), \\
u\left(t, c_{5}\right)=\operatorname{sign}\left(y\left(t, c_{5}\right) B(t)\right) r, \quad t \in[-1,0],\left|c_{5}\right|<\sqrt{1-r}\left(\text { or } c_{5} \in R\right) .
\end{aligned}
$$

If $r>1$, then

$$
\begin{aligned}
y^{0}=-1, \quad x\left(t, c_{6}\right) & =-c_{6} \int_{t}^{0} B^{2}(s) d s, \quad y\left(t, c_{6}\right)=c_{6}, \\
u\left(t, c_{6}\right) & =c_{6} B(t), \quad t \in[-1,0], c_{6} \in[1, r] \cup[-r,-1] .
\end{aligned}
$$

All trajectories meet at the point $(0,0)$ but hypothesis $\left(\mathrm{H}^{\prime}\right)$ is fulfilled.

We form the following canonical spray of flights for the case $r=1$ (we proceed analogously in the other cases):

$$
\begin{gathered}
\begin{array}{c}
\Sigma_{0}^{*}: y^{0}\left(\sigma_{0}\right)=\sigma_{0}, \quad x\left(t, \sigma_{0}\right)=0, \quad y\left(t, \sigma_{0}\right)=0, \quad u\left(t, \sigma_{0}\right)=0, \\
t \in[-1, \pi], \sigma_{0} \in(-1,0),
\end{array} \\
\begin{array}{r}
\Sigma_{11}^{*}: y^{0}=-1, \quad x(t, \sigma)=\sigma_{11} \sin t, \quad y(t, \sigma)=\sigma_{11} \cos t, \\
u(t, \sigma)=\sigma_{11} \cos t, \quad t \in[0, \pi], \sigma_{11}=1-\sigma^{2}, \sigma \in(-1,1), \\
\Sigma_{1 k j}^{*}: y^{0}=-1, \quad x(t, \sigma)=-\sigma_{11} \int_{t}^{0} B^{2}(s) d s, \quad y(t, \sigma)=\sigma_{11}, \\
u(t, \sigma)=\sigma_{11} B(t), \quad t \in I_{k j}, k=0,1, \ldots, j=1,2,3, \\
\Sigma_{21}^{*}: y^{0}=-1, \quad x(t, \sigma)=\sigma_{21} \sin t, \quad y(t, \sigma)=\sigma_{21} \cos t, \\
u(t, \sigma)=\sigma_{21} \cos t, \quad t \in[0, \pi], \sigma_{21}=-1+\sigma^{2}, \sigma \in(-1,1),
\end{array}
\end{gathered}
$$




$$
\begin{aligned}
& \Sigma_{2 k j}^{*}: y^{0}=-1, \quad x(t, \sigma)=-\sigma_{21} \int_{t}^{0} B^{2}(s) d s, \quad y(t, \sigma)=\sigma_{21}, \\
& u(t, \sigma)=\sigma_{21} B(t), \quad t \in I_{k j}, k=0,1, \ldots, j=1,2,3, \\
& \Sigma_{31}^{*}: y^{0}=-1, \quad x\left(t, \sigma_{31}\right)=\operatorname{sign} \sigma_{31}(-t+\pi), \\
& y\left(t, \sigma_{31}\right)=\operatorname{sign} \sigma_{31}\left(\frac{1}{2} t^{2}+\pi t+\sigma_{31} \cos d-\frac{1}{2} d^{2}-\pi d\right), \\
& u\left(t, \sigma_{31}\right)=-\operatorname{sign} \sigma_{31}, \quad t \in\left[\pi-t\left(\sigma_{31}\right), \pi\right] \text {, } \\
& \sigma_{31} \in(-\pi / 2,-1) \cup(1, \pi / 2), d=\pi-t\left(\sigma_{31}\right), \\
& \Sigma_{32}^{*}: y^{0}=-1, \quad x\left(t, \sigma_{31}\right)=\operatorname{sign} \sigma_{31} \sigma_{31} \sin t, \\
& y\left(t, \sigma_{31}\right)=\operatorname{sign} \sigma_{31} \sigma_{31} \cos t, \quad u\left(t, \sigma_{31}\right)=\operatorname{sign} \sigma_{31} \sigma_{31} \cos t, \\
& t \in\left[t\left(\sigma_{31}\right), \pi-t\left(\sigma_{31}\right)\right], \\
& \Sigma_{33}^{*}: y^{0}=-1, \quad x\left(t, \sigma_{31}\right)=\operatorname{sign} \sigma_{31} t, \\
& y\left(t, \sigma_{31}\right)=\operatorname{sign} \sigma_{31}\left(-\frac{1}{2} t^{2}+\sigma_{31} \cos t\left(\sigma_{31}\right)+t^{2}\left(\sigma_{31}\right) / 2\right), \\
& u\left(t, \sigma_{31}\right)=\operatorname{sign} \sigma_{31}, \quad t \in\left[0, t\left(\sigma_{31}\right)\right], \\
& \Sigma_{3 k j}^{*}: y^{0}=-1, \quad x\left(t, \sigma_{31}\right)=-\operatorname{sign} y\left(t, \sigma_{31}\right) \int_{t}^{0} B^{2}(s) d s, \\
& y\left(t, \sigma_{31}\right)=\operatorname{sign} \sigma_{31}\left(\sigma_{31} \cos t\left(\sigma_{31}\right)+t^{2}\left(\sigma_{31}\right) / 2\right), \\
& u\left(t, \sigma_{31}\right)=\operatorname{sign}\left(y\left(t, \sigma_{31}\right) B(t)\right), \quad t \in I_{k j}, k=0,1, \ldots, j=1,2,3 .
\end{aligned}
$$

We join the sprays of flights in three distinguished canonical chains of flights:

$$
\begin{aligned}
& \Sigma_{11}^{*}, \Sigma_{101}^{*}, \Sigma_{102}^{*}, \ldots, \\
& \Sigma_{21}^{*}, \Sigma_{201}^{*}, \Sigma_{202}^{*}, \ldots, \\
& \Sigma_{31}^{*}, \Sigma_{32}^{*}, \Sigma_{33}^{*}, \Sigma_{301}^{*}, \Sigma_{302}^{*}, \ldots
\end{aligned}
$$

These chains, along with $\Sigma_{0}^{*}$, form a concourse of flights (in the sense of $\S 4$ ).

Thus the pair $x(t)=0, u(t)=0, t \in[-1, \pi]$, affords (44) a strong relative minimum.

REMARK. We would like to stress that the second example cannot be solved by the construction from $\S 3$.

\section{REFERENCES}

1. C. Carathéodory, Variationsrechnung und partielle differential Gleichungen erster ordnung, Teubner, Leipzig, 1935.

2. C. Castaing and M. Valadier, Convex analysis and measurable multifunctions, Lecture Notes in Math., vol. 580, Springer, 1977.

3. F. H. Clarke, Generalized gradients and applications, Trans. Amer. Math. Soc. 205 (1975), 247-262.

4. _ Necessary conditions for a general control problem, Proc. Sympos. Calculus of Variations and Optimal Control (D. L. Russel, ed.), Academic Press, New York, 1976.

5. __ Extremal arcs and extended Hamiltonian systems, Trans. Amer. Math. Soc. 231 (1977), 349-367.

6. __ Optimization and nonsmooth analysis, Wiley, 1983.

7. F. H. Clarke and R. B. Vinter, Regularity properties of solutions to the basic problem in the calculus of variations, Trans. Amer. Math . Soc. 289 (1985), 73-98.

8. P. A. Griffiths, Exterior differential systems and the calculus of variations, Birkhäuser, 1983.

9. M. Morse, The calculus of variations in the large, Amer. Math. Soc. Colloq. Publ., vol. 18, Amer. Math. Soc., Providence, R.I., 1934. 
10. A. Nowakowski, Sufficient conditions for a strong relative minimum in an optimal control problem, J. Optim. Theory Appl. 50 (1986), 129-147.

11. R. T. Rockafellar, Existence theorems for general problems of Bolza and Lagrange, Adv. in Math. 15 (1975), 312-333.

12. L. C. Young, Lectures on the calculus of variations and optimal control theory, Saunders, Philadelphia, Pa., 1969.

13. V. Zeidan, Sufficient conditions for the generalized problem of Bolza, Trans. Amer. Math. Soc. 275 (1983), 561-586.

Institute of MAthematics, Łódź University, Lódź, Poland 